\title{
THE CARNEGIE HUBBLE PROGRAM: THE INFRARED LEAVITT LAW IN IC 1613
}

\author{
Victoria Scowcroft ${ }^{1}$, Wendy L. Freedman ${ }^{1}$, Barry F. Madore ${ }^{1}$, Andrew J. Monson ${ }^{1}$, S. E. Persson ${ }^{1}$, \\ Mark Seibert ${ }^{1}, J_{\text {Ane R. RigbY }}^{2}$, and Jason Melbourne ${ }^{3}$ \\ ${ }^{1}$ Observatories of the Carnegie Institution for Science, 813 Santa Barbara Street, Pasadena, CA 91101, USA; vs@ obs.carnegiescience.edu, \\ wendy@obs.carnegiescience.edu, barry@obs.carnegiescience.edu, amonson@obs.carnegiescience.edu, \\ persson@obs.carnegiescience.edu, mseibert@obs.carnegiescience.edu \\ ${ }^{2}$ Observational Cosmology Laboratory, NASA Goddard Space Flight Center, Greenbelt, MD 20771, USA; jane.r.rigby @ nasa.gov \\ ${ }^{3}$ Caltech Optical Observatories, Division of Physics, Mathematics and Astronomy, Mail Stop 301-17, California Institute of Technology, \\ Pasadena, CA 91125, USA; jmel@ caltech.edu \\ Received 2013 January 23; accepted 2013 June 21; published 2013 July 31
}

\begin{abstract}
We have observed the dwarf galaxy IC 1613, at multiple epochs in the mid-infrared using Spitzer and contemporaneously in the near-infrared using the new FourStar near-infrared camera on Magellan. We have constructed Cepheid period-luminosity relations in the $J, H, K_{s}$, [3.6] and [4.5] bands and have used the run of their apparent distance moduli as a function of wavelength to derive the line-of-sight reddening and distance to IC 1613. Using a nine-band fit, we find $E(B-V)=0.05 \pm 0.01 \mathrm{mag}$ and an extinction-corrected distance modulus of $\mu_{0}=24.29 \pm 0.03_{\text {statistical }} \pm 0.03_{\text {systematic }}$ mag. By comparing our multi-band and [3.6] distance moduli to results from the tip of the red giant branch and red clump distance indicators, we find that metallicity has no measurable effect on Cepheid distances at $3.6 \mu \mathrm{m}$ in the metallicity range $-1.0 \leqslant[\mathrm{Fe} / \mathrm{H}] \leqslant 0.2$, hence derivations of the Hubble constant at this wavelength require no correction for metallicity.
\end{abstract}

Key words: galaxies: distances and redshifts - galaxies: individual (IC 1613) - infrared: galaxies - infrared: stars - stars: variables: Cepheids

Online-only material: color figures

\section{INTRODUCTION}

The stated aim of the Carnegie Hubble Program (CHP) is to measure the Hubble constant to an accuracy of $2 \%$ using data from the Warm Spitzer mission, and future data from James Webb Space Telescope and Gaia (see Freedman et al. 2011 for a summary). The calibration of the CHP distance scale is based on mid-infrared observations of Cepheids in the Milky Way (MW) and Local Group galaxies. The distances to individual galaxies are measured by comparing the period-luminosity (PL) relations of their Cepheid populations to that of the MW. In this paper we establish a precise and accurate distance to the Local Group dwarf galaxy IC 1613 using its known population of Cepheids.

Although the slope of the PL relation at a given wavelength is not observed to vary from galaxy to galaxy (Pietrzyński et al. 2006), the sensitivity of the zero-point to various factors is still being debated (e.g., Storm et al. 2011). For example, there has been much discussion over the last few decades regarding the sensitivity of the PL zero-point to metallicity (see Romaniello et al. 2008 for an overview of recent measurements). To test for such an effect we can either observe a galaxy with a known metallicity gradient (as first suggested by Freedman \& Madore 1990 using M31, and later applied to M101 by Kennicutt et al. 1998 and M33 by Scowcroft et al. 2009), or look at a selection of galaxies of different metallicities with an independent distance indicator (e.g., Lee et al. 1993; Sakai et al. 2004).

With $[\mathrm{Fe} / \mathrm{H}] \simeq-1$ (Dolphin et al. 2001) IC 1613 is more metal-poor than the Small Magellanic Cloud, making it extremely useful in setting the low-metallicity calibration of the PL relation. In this paper we compare the distance obtained from the PL relation in the near- and mid-infrared with that measured from the tip of the red giant branch (TRGB) method. Theory suggests that the effect of metallicity in the mid-infrared will be negligible (e.g., McGonegal et al. 1982); however, this has yet to be demonstrated conclusively. The uncertainty in the effect of metallicity on Cepheid magnitudes is one of the dominant systematics still remaining in the extragalactic distance scale. The present test with IC 1613, along with the other metallicity tests described in Freedman et al. (2011) allow us to measure the size and sense of the effect.

IC 1613 was discovered by Wolf (1906). It is a type IB(s)m dwarf irregular galaxy in the Local Group (Sandage 1971; de Vaucouleurs et al. 1991), situated at high galactic latitude at a consensus distance of $736 \pm 49 \mathrm{kpc}^{4}{ }^{4}$ This converts to a true distance modulus of $\mu=24.33 \pm 0.07 \mathrm{mag}$, which is slightly closer than M31. IC 1613 is highly resolved and its position above the plane of the MW results in low foreground extinction. As a dwarf galaxy, IC 1613 should have low internal extinction (see below), it is an ideal system for which to measure a distance, as well as to test and compare different distance indicators.

Studies of the Cepheids in IC 1613 began with Baade (1963), and were followed up by Sandage (1971), who completed the work begun by Baade over 40 years before. Baade chose to observe IC 1613 for the same reasons as we do today: a resolved stellar population and low internal extinction. (Baade deduced the latter from the fact that many background galaxies were visible through the main body of IC 1613.) The low extinction meant that any dispersion in the PL relation would most probably be due to effects intrinsic to the Cepheids themselves (i.e., temperature), rather than differential reddening, say. Sandage (and Baade) found an anomalously and significantly shallower slope of the PL relation $(-1.52$ versus -2.85 in the blue, the latter established for Local Group galaxies). However, the observations were compromised by calibration issues and

\footnotetext{
4 Average distance based on 57 measurements in NED: http://ned.ipac.caltech.edu/cgi-bin/nDistance?name $=\mathrm{IC}+1613$.
} 
Table 1

FourStar Observations of IC 1613

\begin{tabular}{lccccc}
\hline \hline Date & Band & Exposure Time & $N_{\text {dither }}$ & $N_{\text {coadd }}$ & $\begin{array}{c}\text { Total Exposure } \\
(\mathrm{s})\end{array}$ \\
\hline 2011 Sep 9 & $J$ & 20.38 & 9 & 2 & 367 \\
MJD = 55811 & $H$ & 8.733 & 9 & 4 & 314 \\
& $K_{s}$ & 14.56 & 9 & 2 & 262 \\
\hline 2011 Oct 4 & $J$ & 20.38 & 9 & 2 & 376 \\
MJD = 55838 & $H$ & 8.733 & 9 & 4 & 314 \\
& $K_{s}$ & 14.56 & 9 & 2 & 262 \\
\hline 2011 Nov 3 & $J$ & 20.38 & 9 & 2 & 367 \\
MJD = 55868 & $H$ & 5.822 & 9 & 6 & 314 \\
& $K_{s}$ & 5.822 & 9 & 6 & 314 \\
\hline
\end{tabular}

dominated by small-number statistics. Later work by Freedman (1988) resolved the issue, showing that the slope of the PL relation in the visible did not change between galaxies.

Ours is not the first test of the Cepheid metallicity effect using IC 1613. Lee et al. (1993) compared TRGB distances of several local group galaxies to those from Cepheids and RR Lyrae stars and found no significant trend in $\Delta \mu$ with $[\mathrm{Fe} / \mathrm{H}]$ in the $I$ band. Udalski et al. (2001) found 134 Cepheids during the Optical Gravitational Lensing Experiment (OGLE) survey of this galaxy. They compared the $V, I$ and Wesenheit parameter $W_{V I}$ Cepheid distances to the TRGB distance and again found no metallicity effect at those wavelengths. We are repeating their test much further redward with FourStar data in the nearinfrared $\left(J, H\right.$ and $\left.K_{S}\right)$ and Infrared Array Camera (IRAC) data in the mid-infrared (3.6 and $4.5 \mu \mathrm{m})$. The dispersion of the Leavitt law at this wavelength is minimized, not just by the reduction in differential reddening, but because the amplitudes of the Cepheid light curves and the width of the instability strip are minimized (Madore \& Freedman 2012).

A preliminary measure of the mid-infrared PL relation in IC 1613 was made by Freedman et al. (2009, hereafter F09) who searched for Cepheids in archival Spitzer data. They found six Cepheids in the cold-mission data and presented PL relations in the 3.6 and $4.5 \mu \mathrm{m}$ bands. We compare our results to previous work in Section 4.

\section{OBSERVATIONS, DATA REDUCTION, AND CALIBRATION}

\subsection{FourStar: $J, H$, and $K_{s}$}

IC 1613 was observed on three nights using the recently commissioned FourStar wide-field, near-infrared camera (Persson et al. 2013) on the Magellan Baade $6.5 \mathrm{~m}$ telescope at Las Campanas. Table 1 contains the dates and exposure details. IC 1613 virtually fills the $10.8 \times 10.8$ arcmin field of view so the sky background was determined by imaging an adjacent (sparse) field before and after each IC 1613 dither sequence. The same individual exposure times were used but a different number of co-adds and dithers were used to save on overhead. Sources were detected and masked in both the IC 1613 and sky frames. For each IC 1613 frame the nearest nine (in time) sky frames were combined using the unmasked region in common, then scaled to and subtracted from the on-target frames. The IC 1613 frames were then combined using an average with sigma-clipping and input rejection masks. This procedure is not expected to produce either flat or sky subtracted frames with zero offset. However, because the stellar photometry uses local sky measurements, the systematic errors due to offset levels should be negligible.
Table 2

IRAC Observations of IC 1613

\begin{tabular}{lccc}
\hline \hline Date & Average HMJD & Block & Epoch \\
\hline 2010 Jan 26 & 55222.16 & 1 & 1 \\
2010 Feb 6 & 55234.01 & 1 & 2 \\
2010 Feb 14 & 55241.04 & 1 & 3 \\
2010 Feb 25 & 55252.41 & 1 & 4 \\
\hline 2010 Aug 20 & 55428.68 & 2 & 5 \\
2010 Aug 28 & 55436.73 & 2 & 6 \\
2010 Sep 7 & 55446.82 & 2 & 7 \\
2010 Sep 17 & 55456.03 & 2 & 8 \\
\hline 2011 Feb 3 & 55595.95 & 3 & 9 \\
2011 Feb 13 & 55605.14 & 3 & 10 \\
2011 Feb 23 & 55615.08 & 3 & 12 \\
2011 Mar 5 & 55625.57 & 3 & \\
\hline
\end{tabular}

The photometry of the FourStar data was performed with DAOPHOT (Stetson 1987). For each field we identified stars to a signal-to-noise ratio $\mathrm{S} / \mathrm{N} \sim 3$. We generated a model of the point spread function (PSF) across the field from 100 isolated bright stars. We then performed PSF fitting photometry with ALLSTAR allowing the PSF model to vary linearly with $x$ and $y$ across the field.

To determine the precision of the photometry we added 650,000 artificial stars across each mosaic image. Stars were laid down in a grid with spatial offsets between stars of 40 pixels $(10,000$ stars at a time) so as to not increase the crowding. The process was repeated with different grid locations until the library of artificial stars was accumulated. We recovered the positions and photometry of the artificial stars by rerunning the same DAOPHOT routines used for the actual photometry. We achieved better than $10 \%$ precision for stars brighter than $J \sim 21.0, H \sim 19.5$, and $K_{s} \sim 18.5 \mathrm{mag}$. At the high luminosity end the precision is better than $3 \%$.

Photometric zero-points were determined for each epoch by matching 18 bright, but unsaturated, stars in the field of IC 1613 to the Two Micron All Sky Survey (2MASS; Skrutskie et al. 2006). The uncertainties in the zero-points were found to be \pm 0.015 mag or better. However, the scatter around the zero-point for the third epoch of data was about four times larger than for the first two epochs. The larger third epoch scatter was determined to have been caused by excellent seeing. Stellar profiles were $\sim 0$.'3 FWHM, causing the PSF to be undersampled. We applied a smoothing kernel of 1.5 pixels across the mosaics and reran the photometry. This reduced the scatter between 2MASS and FourStar for that epoch to levels comparable to the first epoch. It also reduced the scatter between the FourStar photometry across all three epochs. It did not, however, change the zeropoint significantly, viz., less than 0.005 mag.

\subsection{Spitzer IRAC: 3.6 and $4.5 \mu \mathrm{m}$}

The mid-infrared observations presented here were taken as part of the Warm Spitzer Program PID 61001. We observed in both the $3.6 \mu \mathrm{m}$ and $4.5 \mu \mathrm{m}$ channels with a frame time of $30 \mathrm{~s}$. The galaxy was observed 12 times over 15 months between 2010 January 26 and 2011 March 5. The observations were split into three blocks of roughly one month each and were spaced approximately evenly over that time. The dates of the observations are given in Table 2.

The IRAC camera on Spitzer has two operating channels: the $3.6 \mu \mathrm{m}$ detector observes one field and the $4.5 \mu \mathrm{m}$ detector simultaneously observes a closely adjacent field. At any given 


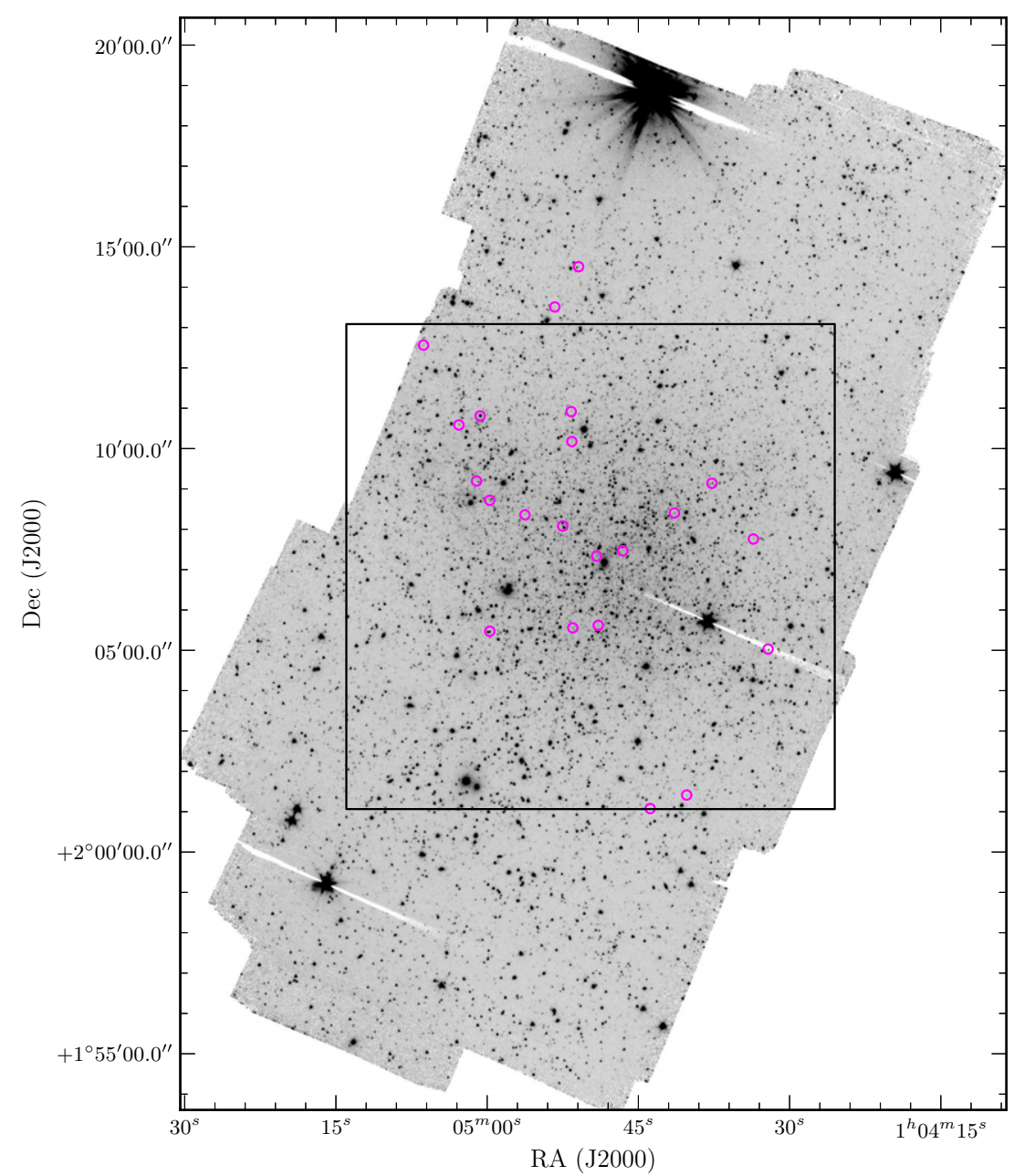

Figure 1. IRAC $3.6 \mu \mathrm{m}$ science mosaic. The central third is covered in all epochs. The black box shows the region observed with FourStar. Magenta circles denote the positions of the Cepheids. Orientation: north is up, east is left.

(A color version of this figure is available in the online journal.)

time one of the detectors will be centered on the target position and the other will be offset. When IRAC takes an exposure it can record both fields simultaneously. This is an advantage in programs such as ours, where a whole galaxy is to be surveyed, as it can cut down the total observation time needed to cover the field in both bands.

However, as the year progresses, Spitzer rotates about its axis so the position of the off-target field rotates around the on-target field. This means that in two thirds of our observations, the $3.6 \mu \mathrm{m}$ off-target observations are to the SE of the main field and in one third they are to the NW (and vice versa for the $4.5 \mu \mathrm{m}$ observations). Therefore, any objects that were not in the "main" field were imaged in only four or eight epochs, rather than all twelve. Note however, that all of our detected Cepheids are in the portion of the image covered by all twelve epochs.

Each observation produced two offset maps, containing the galaxy and its surrounding area, covering approximately $0.15 \times 0.10 \mathrm{deg}$, with a subset roughly $0.10 \times 0.10 \mathrm{deg}$ covered by both channels.

\subsubsection{IRAC Mosaic Creation}

The data were analyzed in two ways: a time-resolved analysis using one mosaic per channel per epoch of observation, and an averaged analysis using a single mosaic per channel, comprising all of the time domain data for that wavelength. In addition to these mosaics, a "master" mosaic was built from all the frames in both channels (1944 frames) and used to determine accurate positions for all the stars in the field. The procedures are described in Section 2.2.5.

\subsubsection{Time-averaged Mosaics}

The data from all epochs were stacked into two mosaics (one per channel) using MOPEX (Makovoz \& Khan 2005). Each mosaic comprises 960 individual basic calibrated data frames. Because of the rotation of the telescope, as described in Section 2, the exposure time coverage within the mosaic is not uniform. The region containing the galaxy has an average coverage of approximately 45 minutes pixel ${ }^{-1}$ in each channel, and has three times the coverage of the cold mission data described in F09. These images will be referred to as the "science mosaics." The $3.6 \mu \mathrm{m}$ science mosaic is shown in Figure 1; the $4.5 \mu \mathrm{m}$ mosaic covers the same area. Approximately the central third of the image has the full 12-epoch coverage, with the outer thirds having coverage at either four or eight epochs.

Due to the large (1".2) pixel scale of IRAC, stellar profiles are badly undersampled in single IRAC frames. However, making use of the large number of observations at each spatial position (960) allowed us to resample the images to achieve higher resolution. The mosaics were created using a pixel scale of 0'75. Other resolutions were tested but 0.75 pixels delivered 
the best sampled PSF and thus smooth, well-sampled profiles for the stars.

Finally, the science mosaics were converted from $\mathrm{MJy} \mathrm{sr}^{-1}$ to data counts using the conversion factors and exposure times in the image headers. The conversion was performed so that ALLFRAME could give a correct estimation of the magnitude uncertainties.

When creating mosaics, MOPEX preserves the fluxes in the original pixels. This means that the variations in the Cepheid fluxes are not truly lost, they are just averaged over. Although the amplitudes of Cepheids in the mid-infrared can reach 0.6 mag, the average of 12 phase points drawn randomly from the light curve will give a good approximation of the mean flux. For example, if we consider a Cepheid with a mid-infrared amplitude of 0.4 mag (typical of the Cepheids in our study), then the average of 12 random observations will have an uncertainty of 0.03 mag (details of this calculation are in the appendix of Scowcroft et al. 2011). It makes no difference to the Cepheids whether we average these points before or after photometry so long as flux is conserved. However, if we stack the twelve images first then we achieve a higher $\mathrm{S} / \mathrm{N}$ image and can detect fainter stars than if we examined single images. Hence, mosaicking the time-resolved images in a way that preserves flux will give us a good value for the average flux of the Cepheid.

\subsubsection{Single-epoch Mosaics}

The single-epoch mosaics were created similarly to the timeaveraged mosaics. Each one was made from 81 images and was resampled to a pixel scale of $0{ }^{\prime \prime} 6^{5} .^{5}$ The images were converted from $\mathrm{MJy} \mathrm{sr}^{-1}$ to counts using the conversion factors and exposure times in the image headers. Again, the dither pattern meant that the mosaics were not uniformly exposed, but now had typical integration times of 5 minutes pixel $^{-1}$. Note that the single-epoch mosaics are shallower than the data used by F09. Consequently, the time-averaged mosaics were used for the final photometry, while the time-resolved data were used only to confirm that the stars identified as Cepheids were truly variable.

\subsubsection{Correction Mosaics}

In addition to the science mosaics, MOPEX was used to make "correction" mosaics. These are made identically to the science mosaics, mosaicking the location-dependent correction images provided by the Spitzer Science Center ${ }^{6}$ in the same geometrical pattern used for the science mosaics. The correction mosaics are necessary as IRAC is not uniformly sensitive over its entire field of view. The non-uniform coverage depth of our science mosaics can further exacerbate the problem. Inspection of the correction mosaics showed that the residual location-dependent effect had mean values of approximately $2 \%$ in both channels, and would reach as high as $7 \%$ at $3.6 \mu \mathrm{m}$ and $10 \%$ at $4.5 \mu \mathrm{m}$ in particularly non-uniform regions, if left uncorrected.

\subsubsection{DAOPHOT and ALLFRAME Reduction}

The photometry was performed using the DAOPHOT and ALlFRAME packages (Stetson 1987, 1994). The mosaics were run through DAOPHOT to detect the stars and create a PSF model. The detected objects were subtracted and the resulting

\footnotetext{
5 As the single-epoch mosaics were shallow we did not perform the same tests to find the optimum image scale, instead we used the default value.

6 Details of the location-dependent photometric correction are given in Section 4.5 of the IRAC instrument handbook.
}

frame processed again to detect the remaining objects. The PSF model was created using $\sim 100$ stars in each mosaic. The final photometry was done using ALLFRAME. ALLFRAME is preferred over ALLSTAR in studies where there are multiple frames of the same field as it will produce a master detection list. When the photometry is done using the positions in this list, rather than remeasuring the positions from each frame, the number of free parameters in the PSF fit are reduced. This significantly reduces the uncertainty in the final magnitude, and allows better de-blending of close sources. A detailed description of the process can be found in Stetson (1994). The master detection list was generated by running DAOPHOT on the master frame. The coordinate transformations between this frame and all the other mosaics were determined, then were input to ALLFRAME to produce the instrumental photometry.

Artificial star tests were performed on the IRAC images to test the precision of the photometry. Following the methodology set out by Stetson \& Harris (1988), ADDSTAR was used to add 10,000 stars to the 3.6 and $4.5 \mu \mathrm{m}$ images, 100 stars at a time so as not to significantly increase the level of crowding. We achieve $10 \%$ precision for stars brighter than $19.2 \mathrm{mag}(3.6 \mu \mathrm{m})$ and $19.3 \mathrm{mag}(4.5 \mu \mathrm{m})$. We also find that crowding does not significantly affect the photometry - the median difference between the input and output magnitudes for the artificial stars was less than $0.01 \mathrm{mag}$ in both channels.

\subsubsection{Calibration}

All the Spitzer photometry in the CHP is set to be on the standard system defined by Reach et al. (2005, hereafter R05). As we used mosaics for our photometry rather than single images we did not need to apply a pixel-phase correction. Any effects due to pixel phase should be adequately averaged over by the dithering and mosaicking. Placing the instrumental magnitudes on the R05 system was achieved by using the PSF stars in the science mosaics as local standards.

The PSF stars in the flux-units versions of the science mosaics (i.e., after mosaicking but before conversion to counts) were each measured using the РНОТ aperture photometry routine in IRAF. $^{7}$ The zmag parameter in PHOT was set for each channel such that the procedure would output calibrated magnitudes.

Each of the stars was measured in a 3".6 radius aperture and sky annulus from 3 .' 6 to 8 ". 4 , corresponding to the standard aperture set of 3, 3, 7 native IRAC pixels. To convert this to the $12^{\prime \prime}$ radius (10 native pixels) standard aperture used by R05, the latest warm mission aperture corrections of 1.128 and 1.127 in channels one and two, respectively (Spitzer Science Center 2012, private communication) were applied to the measured fluxes.

The ALLFRAME photometry was calibrated by finding the offset between it and the corrected aperture magnitudes described above and correcting each star in the field accordingly. Finally, the location-dependent correction was applied by measuring the pixel values in the correction mosaic at the positions of each star in our catalog and multiplying the flux of each star by this value.

\section{THE CEPHEID POPULATION OF IC 1613}

Cepheids were identified by matching to the catalog from the OGLE study (Udalski et al. 2001). We also identified V22 from Sandage (1971), a long-period Cepheid which was not

\footnotetext{
7 IRAF is distributed by the National Optical Astronomy Observatory, which is operated by the Association of Universities for Research in Astronomy (AURA) under cooperative agreement with the National Science Foundation.
} 
Table 3

Near- and Mid-infrared Mean Magnitudes of Cepheids Found in IC 1613

\begin{tabular}{|c|c|c|c|c|c|c|c|c|c|c|c|c|c|c|}
\hline OGLE ID & $\begin{array}{l}\text { Period } \\
\text { (days) }\end{array}$ & $\begin{array}{c}\text { R.A. } \\
\text { (hh:mm:ss) }\end{array}$ & $\begin{array}{c}\text { Decl. } \\
\text { (dd:mm:ss) }\end{array}$ & $\begin{array}{c}J \\
(\mathrm{mag})\end{array}$ & $\begin{array}{c}\sigma_{J} \\
(\mathrm{mag})\end{array}$ & $\begin{array}{c}H \\
(\mathrm{mag})\end{array}$ & $\begin{array}{c}\sigma_{H} \\
(\mathrm{mag})\end{array}$ & $\begin{array}{c}K_{s} \\
(\mathrm{mag})\end{array}$ & $\begin{array}{c}\sigma_{K_{S}} \\
(\mathrm{mag})\end{array}$ & $\begin{array}{l}{[3.6]} \\
(\mathrm{mag})\end{array}$ & $\begin{array}{l}\sigma_{[3.6]} \\
(\mathrm{mag})\end{array}$ & $\begin{array}{l}{[4.5]} \\
(\mathrm{mag})\end{array}$ & $\begin{array}{l}\sigma_{[4.5]} \\
(\mathrm{mag})\end{array}$ & S71 \\
\hline $\mathrm{V} 22^{\mathrm{a}, \mathrm{b}}$ & 123.880 & 1:05:00.701 & $+02: 10: 48.60$ & 15.878 & 0.004 & 15.644 & 0.003 & 15.454 & 0.002 & 15.369 & 0.009 & 15.457 & 0.017 & V22 \\
\hline $11446^{\mathrm{a}}$ & 41.630 & $1: 04: 59.740$ & $+02: 05: 28.30$ & 17.139 & 0.012 & 16.970 & 0.009 & 16.866 & 0.007 & 16.600 & 0.014 & 16.610 & 0.018 & V20 \\
\hline 736 & 23.45 & $1: 04: 32.130$ & $+02: 05: 01.90$ & $\ldots$ & $\ldots$ & $\ldots$ & $\ldots$ & $\ldots$ & $\ldots$ & 17.903 & 0.162 & 17.325 & 0.043 & $\mathrm{~V} 2$ \\
\hline $7647^{\mathrm{a}}$ & 16.540 & 1:04:37.700 & $+02: 09: 08.40$ & 18.052 & 0.020 & 17.916 & 0.016 & 17.854 & 0.014 & 17.656 & 0.032 & 17.694 & 0.031 & \\
\hline 13738 & 16.37 & $1: 05: 02.810$ & $+02: 10: 35.10$ & 18.440 & 0.027 & 18.210 & 0.019 & 18.025 & 0.014 & 18.040 & 0.021 & 18.199 & 0.021 & V18 \\
\hline $7664^{\mathrm{a}}$ & 10.450 & $1: 04: 41.420$ & $+02: 08: 24.20$ & 19.038 & 0.042 & 18.825 & 0.032 & 18.726 & 0.027 & 18.585 & 0.025 & 18.637 & 0.039 & V16 \\
\hline $926^{\mathrm{a}}$ & 9.402 & $1: 04: 33.590$ & $+02: 07: 45.60$ & 19.016 & 0.041 & 18.838 & 0.029 & 18.784 & 0.025 & 18.597 & 0.029 & 18.541 & 0.045 & V06 \\
\hline 11589 & 8.409 & $1: 04: 51.510$ & $+02: 05: 33.50$ & 19.490 & 0.075 & 19.291 & 0.050 & 19.245 & 0.043 & 18.404 & 0.056 & 18.504 & 0.0585 & V34 \\
\hline 13808 & 7.557 & 1:04:59.740 & $+02: 08: 43.10$ & 19.617 & 0.069 & 19.332 & 0.044 & 19.262 & 0.038 & $\ldots$ & $\ldots$ & $\ldots$ & $\ldots$ & $\ldots$ \\
\hline 13759 & 7.333 & 1:04:52.510 & $+02: 08: 04.80$ & 19.491 & 0.068 & 19.331 & 0.048 & 19.298 & 0.043 & 18.336 & 0.068 & 18.451 & 0.071 & V7 \\
\hline 18905 & 6.766 & 1:05:06.310 & $+02: 12: 33.90$ & 19.734 & 0.050 & 19.481 & 0.038 & 19.441 & 0.033 & 18.989 & 0.079 & 19.310 & 0.104 & \\
\hline $13943^{a}$ & 6.751 & $1: 04: 51.670$ & $+02: 10: 55.00$ & 19.407 & 0.091 & 19.217 & 0.064 & 19.134 & 0.054 & 18.990 & 0.041 & 19.101 & 0.046 & V24 \\
\hline 3732 & 6.669 & $1: 04: 40.210$ & $+02: 01: 24.80$ & $\ldots$ & $\ldots$ & $\ldots$ & $\ldots$ & $\ldots$ & $\ldots$ & 19.250 & 0.041 & 19.117 & 0.080 & V27 \\
\hline 5037 & 6.31 & $1: 04: 49.140$ & $+02: 07: 20.20$ & 20.149 & 0.109 & 19.850 & 0.080 & 19.790 & 0.072 & 19.140 & 0.079 & 18.958 & 0.103 & $\ldots$ \\
\hline 3722 & 5.818 & 1:04:43.830 & $+02: 01: 04.70$ & $\ldots$ & $\ldots$ & $\ldots$ & $\ldots$ & $\ldots$ & $\ldots$ & 19.734 & 0.074 & 19.317 & 0.082 & V26 \\
\hline 13911 & 5.717 & 1:04:51.600 & $+02: 10: 10.50$ & 19.911 & 0.081 & 19.688 & 0.055 & 19.638 & 0.050 & 19.481 & 0.064 & 19.401 & 0.085 & V17 \\
\hline 13780 & 5.58 & 1:04:56.250 & $+02: 08: 21.60$ & 19.965 & 0.087 & 19.717 & 0.060 & 19.684 & 0.053 & 19.501 & 0.058 & 19.407 & 0.082 & V9 \\
\hline 4875 & 5.138 & 1:04:48.980 & $+02: 05: 37.10$ & 19.720 & 0.079 & 19.579 & 0.060 & 19.542 & 0.051 & 19.330 & 0.041 & 19.283 & 0.058 & V14 \\
\hline 15696 & 5.012 & 1:04:50.930 & $+02: 14: 30.60$ & $\ldots$ & $\ldots$ & $\ldots$ & $\ldots$ & $\ldots$ & $\cdots$ & 19.426 & 0.072 & 19.645 & 0.078 & $\cdots$ \\
\hline 15670 & 4.849 & 1:04:53.290 & $+02: 13: 30.60$ & $\ldots$ & $\ldots$ & $\ldots$ & $\ldots$ & $\ldots$ & $\ldots$ & 19.456 & 0.061 & 19.312 & 0.101 & V13 \\
\hline 14287 & 4.365 & 1:05:01.050 & $+02: 09: 11.80$ & 20.484 & 0.104 & 20.252 & 0.079 & 20.202 & 0.070 & 19.582 & 0.091 & 19.692 & 0.134 & $\ldots$ \\
\hline 13784 & 4.045 & 1:04:59.848 & $+01: 53: 10.16$ & $\ldots$ & $\ldots$ & $\ldots$ & $\cdots$ & $\ldots$ & $\cdots$ & 19.216 & 0.047 & 19.385 & 0.075 & V10 \\
\hline 6084 & 3.872 & 1:04:46.550 & $+02: 07: 28.10$ & $\ldots$ & $\cdots$ & $\cdots$ & $\cdots$ & $\cdots$ & $\cdots$ & 19.667 & 0.087 & 19.329 & 0.095 & $\ldots$ \\
\hline
\end{tabular}

Notes.

a Detected by Freedman et al. (2009).

b No OGLE ID.

included in the OGLE catalog, but was included by F09. The Cepheids were initially identified by their positions derived from the science mosaics. The science mosaics were then visually inspected to check for possible nearby contaminants. The light curves generated from the single-epoch mosaics were inspected to check for variability, but this became increasingly difficult at periods below 10 days as the uncertainties on the individual points were comparable to the amplitudes of the light curves.

Thirty-one stars measured at 3.6 and $4.5 \mu \mathrm{m}$ were matched with the OGLE catalog, and of these twenty-two were measured at $J, H$, and $K_{s}$. This is just under $25 \%$ of the original OGLE sample. Unfortunately, the majority of Cepheids in IC 1613 have periods below 6 days. Short period Cepheids are naturally fainter and the majority of the OGLE sample fell below our detection limit.

Several of the Cepheids detected in the IRAC images appeared anomalously bright for their known periods. After visual inspection of both the near- and mid-infrared images the stars were deemed to be blended with unresolved companions and were excluded from all further analysis. After a review of all of the Cepheids, blends were removed and 20 Cepheids remained. The photometry of the final sample of Cepheids is given in Table 3. The near-infrared time series data is given in Tables 4-6.

\subsection{Near-infrared Period-Luminosity Relations}

PL relations were obtained in the $J, H$, and $K_{s}$ bands. In this case we had three epochs that were reduced individually. The magnitudes are weighted means of the three observations of each Cepheid, and the uncertainties are the errors of the weighted mean. The systematic uncertainties in the near-infrared photometric zero-points are $0.017,0.020$ and $0.021 \mathrm{mag}$ in $J, H$, and $K_{s}$, respectively.
Table 4

$J$ Band Time Series Photometry of Cepheids in IC 1613

\begin{tabular}{|c|c|c|c|c|c|c|}
\hline OGLE ID & $\begin{array}{c}J_{1}{ }^{\mathrm{a}} \\
(\mathrm{mag})\end{array}$ & $\begin{array}{c}\sigma_{J_{1}} \\
(\mathrm{mag})\end{array}$ & $\begin{array}{c}J_{2}{ }^{\mathrm{b}} \\
(\mathrm{mag})\end{array}$ & $\begin{array}{c}\sigma_{J_{2}} \\
(\mathrm{mag})\end{array}$ & $\begin{array}{c}J_{3}{ }^{\mathrm{c}} \\
(\mathrm{mag})\end{array}$ & $\begin{array}{c}\sigma_{J_{3}} \\
(\mathrm{mag})\end{array}$ \\
\hline V22 & 15.709 & 0.008 & 15.754 & 0.007 & 16.192 & 0.007 \\
\hline 11446 & 17.079 & 0.014 & 17.205 & 0.057 & 17.397 & 0.028 \\
\hline 736 & $\cdots$ & $\cdots$ & $\cdots$ & $\cdots$ & $\cdots$ & $\cdots$ \\
\hline 7467 & 18.21 & 0.042 & 17.988 & 0.046 & 18.018 & 0.026 \\
\hline 13738 & 18.67 & 0.079 & 18.454 & 0.038 & 18.36 & 0.044 \\
\hline 7664 & 18.813 & 0.079 & 19.153 & 0.07 & 19.13 & 0.07 \\
\hline 926 & 19.011 & 0.057 & 19.02 & 0.083 & 19.023 & 0.085 \\
\hline 11589 & 19.354 & 0.14 & 19.457 & 0.126 & 19.654 & 0.126 \\
\hline 13808 & 19.661 & 0.127 & 19.581 & 0.128 & 19.613 & 0.109 \\
\hline 13759 & 19.497 & 0.13 & 19.459 & 0.113 & 19.519 & 0.111 \\
\hline 18905 & 19.742 & 0.084 & 19.789 & 0.111 & 19.702 & 0.076 \\
\hline 13943 & $\cdots$ & $\cdots$ & 19.407 & 0.091 & $\cdots$ & $\cdots$ \\
\hline 3732 & $\cdots$ & $\ldots$ & $\ldots$ & $\ldots$ & $\cdots$ & $\cdots$ \\
\hline 5037 & 20.056 & 0.18 & 19.975 & 0.16 & 21.408 & 0.268 \\
\hline 3722 & $\ldots$ & $\ldots$ & $\ldots$ & $\ldots$ & $\ldots$ & $\ldots$ \\
\hline 13911 & 20.017 & 0.148 & 19.807 & 0.137 & 19.935 & 0.137 \\
\hline 13780 & 19.957 & 0.175 & 19.849 & 0.137 & 20.12 & 0.146 \\
\hline 4875 & 19.718 & 0.134 & 19.743 & 0.144 & 19.703 & 0.135 \\
\hline 15696 & $\ldots$ & $\ldots$ & $\ldots$ & $\ldots$ & $\ldots$ & $\cdots$ \\
\hline 15670 & $\ldots$ & $\cdots$ & $\cdots$ & $\cdots$ & $\cdots$ & $\cdots$ \\
\hline 14287 & $\cdots$ & $\cdots$ & $\cdots$ & $\cdots$ & $\cdots$ & $\cdots$ \\
\hline 13784 & $\cdots$ & $\cdots$ & $\cdots$ & $\cdots$ & $\cdots$ & $\cdots$ \\
\hline 6084 & $\ldots$ & $\cdots$ & $\cdots$ & $\cdots$ & $\cdots$ & $\cdots$ \\
\hline
\end{tabular}

Notes.

${ }^{\text {a }} \mathrm{MJD}_{1}-2,400,000=55,811$.

${ }^{\mathrm{b}} \mathrm{MJD}_{2}-2,400,000=55,838$.

${ }^{\text {c }} \mathrm{MJD}_{3}-2,400,000=55,868$. 


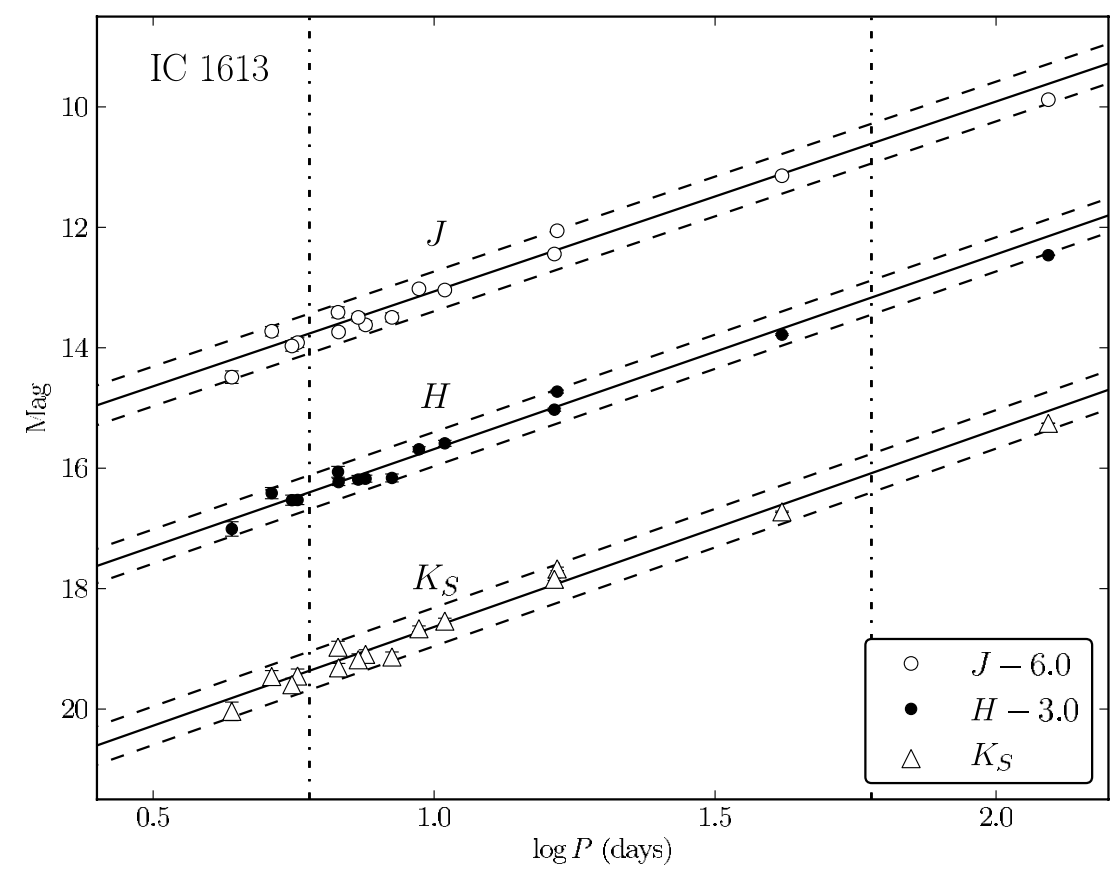

Figure 2. Period-luminosity relations in the $J, H$ and $K_{s}$ bands. The solid lines represent the fitted PL relations; the dashed lines delineate the $\pm 2 \sigma$ width of the instability strip. The vertical dot-dash lines show the period range (6-60 days) used to fit the PL relations.

Table 5

$H$ Band Time Series Photometry of Cepheids in IC 1613

\begin{tabular}{|c|c|c|c|c|c|c|}
\hline OGLE ID & $\begin{array}{c}H_{1}{ }^{\mathrm{a}} \\
(\mathrm{mag})\end{array}$ & $\begin{array}{c}\sigma_{H_{1}} \\
(\mathrm{mag})\end{array}$ & $\begin{array}{c}H_{2}{ }^{\mathrm{b}} \\
(\mathrm{mag})\end{array}$ & $\begin{array}{c}\sigma_{H_{2}} \\
(\mathrm{mag})\end{array}$ & $\begin{array}{c}H_{3}{ }^{\mathrm{c}} \\
(\mathrm{mag})\end{array}$ & $\begin{array}{c}\sigma_{H_{3}} \\
(\mathrm{mag})\end{array}$ \\
\hline V22 & 15.305 & 0.007 & 15.357 & 0.009 & 15.652 & 0.006 \\
\hline 11446 & 16.622 & 0.034 & 16.82 & 0.019 & 16.803 & 0.027 \\
\hline 736 & $\ldots$ & $\ldots$ & . & $\cdots$ & $\cdots$ & $\ldots$ \\
\hline 7467 & 17.844 & 0.049 & 17.687 & 0.041 & 17.681 & 0.043 \\
\hline 13738 & 18.257 & 0.043 & 17.975 & 0.046 & 17.839 & 0.049 \\
\hline 7664 & 18.495 & 0.073 & 18.712 & 0.093 & 18.617 & 0.098 \\
\hline 926 & 18.67 & 0.077 & 18.737 & 0.071 & 18.641 & 0.069 \\
\hline 11589 & 19.085 & 0.104 & 19.179 & 0.115 & 19.252 & 0.13 \\
\hline 13808 & 19.232 & 0.101 & 19.162 & 0.102 & 19.127 & 0.097 \\
\hline 13759 & 19.178 & 0.112 & 19.227 & 0.137 & 19.164 & 0.115 \\
\hline 18905 & 19.328 & 0.133 & 19.177 & 0.091 & 19.227 & 0.087 \\
\hline 13943 & $\ldots$ & $\ldots$ & 19.058 & 0.09 & $\ldots$ & $\cdots$ \\
\hline 3732 & $\cdots$ & $\cdots$ & $\cdots$ & $\cdots$ & $\ldots$ & $\cdots$ \\
\hline 5037 & 19.637 & 0.18 & 19.554 & 0.152 & $\ldots$ & $\cdots$ \\
\hline 3722 & $\ldots$ & $\ldots$ & $\ldots$ & $\ldots$ & $\cdots$ & $\cdots$ \\
\hline 13911 & 19.702 & 0.154 & 19.474 & 0.123 & 19.474 & 0.123 \\
\hline 13780 & 19.511 & 0.122 & 19.448 & 0.156 & 19.67 & 0.176 \\
\hline 4875 & 19.449 & 0.174 & 19.361 & 0.175 & 19.416 & 0.142 \\
\hline 15696 & $\cdots$ & $\cdots$ & $\ldots$ & $\cdots$ & $\cdots$ & $\cdots$ \\
\hline 15670 & $\cdots$ & $\cdots$ & $\cdots$ & $\cdots$ & $\cdots$ & $\cdots$ \\
\hline 14287 & 20.023 & 0.283 & 20.073 & 0.215 & 19.958 & 0.168 \\
\hline 13784 & $\cdots$ & $\cdots$ & $\cdots$ & $\cdots$ & $\cdots$ & $\cdots$ \\
\hline 6084 & $\cdots$ & $\cdots$ & $\ldots$ & $\ldots$ & $\cdots$ & $\cdots$ \\
\hline
\end{tabular}

Notes.

${ }^{\mathrm{a}} \mathrm{MJD}_{1}-2,400,000=55,811$

b $\mathrm{MJD}_{2}-2,400,000=55,838$.

${ }^{\mathrm{c}} \mathrm{MJD}_{3}-2,400,000=55,868$.

The PL relations for the near-infrared $J, H$, and $K_{s}$ bands are shown in Figure 2. Prior to fitting, the magnitudes were converted from the 2MASS system to the LCO photometric system, using the transformations described in Section 4.6 of Carpenter (2001). The LMC PL relations from Table 6 of
Table 6

$K_{S}$ Band Time Series Photometry of Cepheids in IC 1613

\begin{tabular}{|c|c|c|c|c|c|c|}
\hline OGLE ID & $\begin{array}{c}K_{S 1}^{\mathrm{a}} \\
(\mathrm{mag})\end{array}$ & $\begin{array}{c}\sigma_{K_{S 1}} \\
(\mathrm{mag})\end{array}$ & $\begin{array}{l}K_{S 2}{ }^{\mathrm{b}} \\
(\mathrm{mag})\end{array}$ & $\begin{array}{c}\sigma_{K_{S 2}} \\
(\mathrm{mag})\end{array}$ & $\begin{array}{l}K_{S 3}{ }^{\mathrm{c}} \\
(\mathrm{mag})\end{array}$ & $\begin{array}{c}\sigma_{K_{S 3}} \\
(\mathrm{mag})\end{array}$ \\
\hline V22 & 15.159 & 0.005 & 15.189 & 0.006 & 15.532 & 0.007 \\
\hline 11446 & 16.539 & 0.025 & 16.79 & 0.019 & 16.753 & 0.019 \\
\hline 736 & $\ldots$ & $\ldots$ & $\ldots$ & . & $\ldots$ & $\cdots$ \\
\hline 7467 & 17.772 & 0.06 & 17.603 & 0.047 & 17.661 & 0.048 \\
\hline 13738 & 18.161 & 0.048 & 17.841 & 0.041 & 17.741 & 0.028 \\
\hline 7664 & 18.427 & 0.072 & 18.628 & 0.093 & 18.615 & 0.091 \\
\hline 926 & 18.635 & 0.07 & 18.732 & 0.078 & 18.58 & 0.104 \\
\hline 11589 & 19.019 & 0.148 & 19.119 & 0.148 & 19.236 & 0.138 \\
\hline 13808 & 19.147 & 0.218 & 19.082 & 0.112 & 19.064 & 0.116 \\
\hline 13759 & 19.16 & 0.182 & 19.207 & 0.167 & 19.149 & 0.162 \\
\hline 18905 & 19.262 & 0.129 & 19.337 & 0.127 & 19.317 & 0.122 \\
\hline 13943 & $\ldots$ & $\ldots$ & 18.963 & 0.098 & $\ldots$ & $\ldots$ \\
\hline 3732 & $\ldots$ & $\ldots$ & $\ldots$ & $\ldots$ & $\ldots$ & $\ldots$ \\
\hline 5037 & 19.556 & 0.168 & $\ldots$ & $\ldots$ & $\ldots$ & $\cdots$ \\
\hline 3722 & $\ldots$ & $\ldots$ & $\ldots$ & $\ldots$ & $\ldots$ & $\ldots$ \\
\hline 13911 & 19.46 & 0.194 & 19.437 & 0.208 & 19.426 & 0.203 \\
\hline 13780 & 19.566 & 0.183 & 19.637 & 0.187 & 19.56 & 0.183 \\
\hline 4875 & 19.459 & 0.171 & 19.497 & 0.179 & 19.405 & 0.161 \\
\hline 15696 & $\ldots$ & $\ldots$ & $\ldots$ & $\ldots$ & $\ldots$ & $\ldots$ \\
\hline 15670 & $\ldots$ & $\ldots$ & $\ldots$ & $\ldots$ & $\ldots$ & $\ldots$ \\
\hline 14287 & $\ldots$ & $\ldots$ & 19.996 & 0.219 & 20.063 & 0.216 \\
\hline 13784 & $\ldots$ & $\ldots$ & $\ldots$ & $\ldots$ & $\ldots$ & $\ldots$ \\
\hline 6084 & $\ldots$ & $\ldots$ & $\ldots$ & $\ldots$ & $\ldots$ & $\ldots$ \\
\hline
\end{tabular}

Notes.

${ }^{\mathrm{a}} \mathrm{MJD}_{1}-2,400,000=55,811$.

b $\mathrm{MJD}_{2}-2,400,000=55,838$.

${ }^{\mathrm{c}} \mathrm{MJD}_{3}-2,400,000=55,868$.

Persson et al. (2004) are adopted as fiducial and are rewritten in the form

$$
M_{\lambda}=a_{\lambda}(\log P-1.0)+b_{\lambda} .
$$

We fixed the slopes $a_{\lambda}$ to the LMC values and used an unweighted least squares fit to find the zero-points $b_{\lambda}$. 


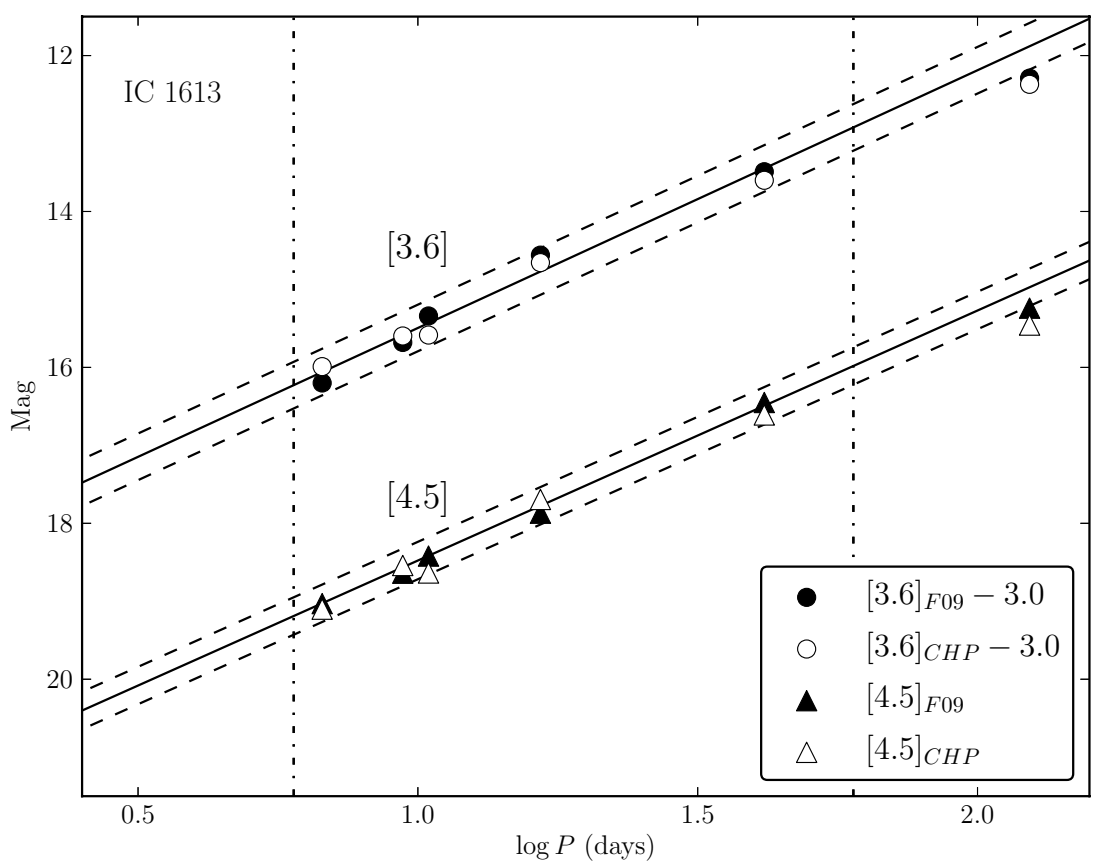

Figure 3. Comparison of photometry from this paper with that of Freedman et al. (2009) for the six Cepheids they detected. The magnitudes are not expected to be identical as theirs are single-epoch observations, while ours are averages over twelve phase points. The filled symbols are the cold mission data, the open symbols are the CHP data. Solid lines depict the PL fit to the CHP data (fixing the slopes to LMC values, using the sample with $6 \leqslant P \leqslant 60$ days) and are indistinguishable from the fits to the cold data. Dashed lines are $\pm 2 \sigma$ around the fits. The vertical dot-dash lines show the period range (6-60 days) used to fit the PL relations.

Table 7

Mid-infrared Period-Luminosity Relation Zero-points for Unblended Cepheids in IC 1613

\begin{tabular}{lccc}
\hline \hline Band & $\begin{array}{c}\text { Zero-point } \\
(\mathrm{mag})\end{array}$ & Standard Deviation & $\begin{array}{c}\mu \\
(\mathrm{mag})\end{array}$ \\
\hline$J$ & $19.07 \pm 0.05$ & 0.206 & $24.35 \pm 0.05^{\mathrm{b}}$ \\
$H$ & $18.69 \pm 0.04$ & 0.165 & $24.30 \pm 0.04^{\mathrm{b}}$ \\
$K_{S}$ & $18.64 \pm 0.05$ & 0.168 & $24.33 \pm 0.05^{\mathrm{b}}$ \\
{$[3.6]$} & $18.51 \pm 0.08$ & 0.307 & $24.31 \pm 0.09^{\mathrm{c}}$ \\
{$[4.5]$} & $18.50 \pm 0.07$ & 0.235 & $24.26 \pm 0.08^{\mathrm{c}}$ \\
\hline
\end{tabular}

Notes.

${ }^{a}$ PL relations took the form $M=a(\log P-1.0)+b$; the $a$ coefficients are taken from Persson et al. (2004) $\left(J, H, K_{S}\right)$ and Scowcroft et al. (2011) ([3.6], [4.5]).

${ }^{\mathrm{b}}$ Distance moduli were calculated using the LMC PL relation zero-points and uncertainties from Persson et al. (2004), and assuming $\mu_{0, \mathrm{LMC}}=18.48$. They have not been corrected for extinction.

${ }^{c}$ Distance moduli were calculated using the MW PL relation zero-points and uncertainties from Monson et al. (2012). They have not been corrected for extinction

The resulting fits are listed in Table 7 . The fits assume $\mu_{0, \mathrm{LMC}}=$ $18.48 \pm 0.03 \mathrm{mag}$, as derived in Monson et al. (2012) and Freedman et al. (2012); this puts them on the same scale as the midinfrared values. The apparent distance moduli derived from the near-infrared PL relations are $24.36 \pm 0.05,24.31 \pm 0.04$ and $24.34 \pm 0.05 \mathrm{mag}$ in $J, H$, and $K_{s}$ respectively.

\subsection{Mid-infrared Period-Luminosity Relations}

The mid-infrared Leavitt laws take the same form as the near-infrared laws given in Equation (1), but in this case the slopes were taken from the Scowcroft et al. (2011) LMC results and the zero-points were derived using an unweighted least-squares fit.

The first comparison we make is with the earlier results of F09. They measured single-phase magnitudes for six IC 1613 Cepheids in data obtained from the Spitzer archive. The resulting
PL relations are plotted in Figure 3, along with the CHP time-averaged magnitudes for the same six Cepheids.

We do not expect the values to be exactly the same; the amplitude of each Cepheid's light curve will change its position relative to the ridge line of the PL relation in the F09 values (and to a much lesser extent in the CHP values depending on the dispersion of the phase points throughout the pulsation cycle). Hence it not necessarily useful to compare the magnitudes of individual stars. However, on average the results should agree, such that we should get the same result when we fit the PL relation to either set of data.

The PL was found by fixing the slope to the values derived from the LMC (Scowcroft et al. 2011) and making an unweighted least-squares fit to the Cepheids with $6 \leqslant P \leqslant$ 60 days. This period range was chosen to match the MW and LMC samples used to define the IRAC PL relations. The fits to the F09 and CHP data are indistinguishable when plotted; the zero-points differ by only $0.02 \mathrm{mag}$, which is much smaller than the formal $1 \sigma$ error on the fitted zero-points $(0.15$ and $0.12 \mathrm{mag}$ in [3.6] and [4.5], respectively).

Finally, we re-fit the PL using the whole sample of Cepheids in the period range 6-60 days. A montage of the $3.6 \mu \mathrm{m}$ images of these Cepheids can be found in Figure 4. The results are given in Table 7, and the relations are plotted in Figure 5. By comparing the calculated zero-points to those in the MW PL relations given in Monson et al. (2012) we find apparent distance moduli of IC 1613 of $24.31 \pm 0.09$ and $24.26 \pm 0.08$ mag using the [3.6] and [4.5] PL relations, respectively. The quoted uncertainties include the error estimates from both the MW and IC 1613 fits.

\subsection{Reddening Corrected Distance Modulus}

We can make use of the broad wavelength coverage of archival observations to derive the total line-of-sight reddening and extinction to the Cepheids in IC 1613. Figure 6 demonstrates 

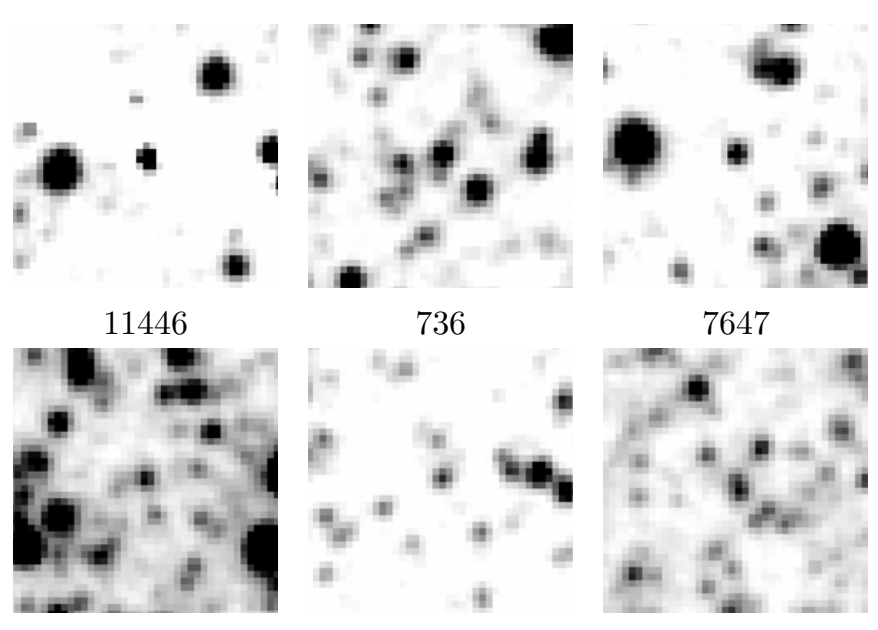

13738
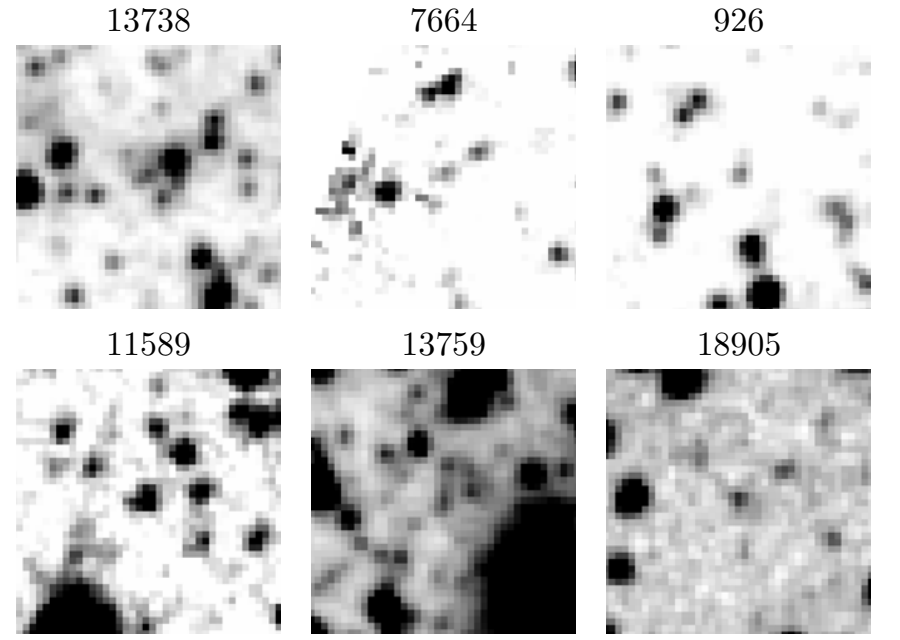

13943
3732

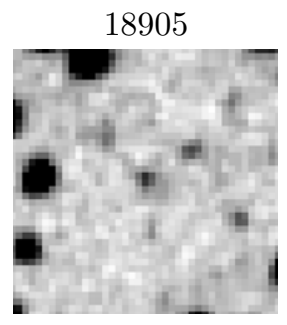

5037

Figure 4. A montage of $3.6 \mu \mathrm{m}$ images of the Cepheids used in the midinfrared PL relation. The Cepheids are the stars in the center of each image. The PSF-subtracted image was examined for every Cepheid and the ones in the final PL relation were cleanly subtracted from the image, showing no evidence of crowding.

the technique. The distance moduli are plotted as a function of inverse wavelength in microns. Three extinction laws-the optical and near-infrared laws from Cardelli et al. (1989) and the mid-infrared law from Indebetouw et al. $(2005)^{8}$ — are combined to fit to the data, assuming $R_{V}=3.1$. The best fit $E(B-V)$ value was found by minimizing the dispersion of the distance moduli around the scaled and shifted extinction law, and was found to be $E(B-V)=0.05 \pm 0.01 \mathrm{mag}$. The top panel in Figure 6 shows the scaled, shifted extinction law with the apparent distance moduli at nine wavelengths; the dashed lines show how the fit changes if $E(B-V)$ is changed by $1 \sigma$. Applying this scaled correction to each of the apparent distance moduli and taking a weighted average results in an absolute distance modulus of $\left\langle\mu_{0}\right\rangle=24.29 \pm 0.03_{\text {stat }} \pm 0.03_{\text {sys }}$ mag. The deviations of the extinction-corrected distances around this value are shown in the bottom panel of Figure 6. The systematic uncertainty comes from the LMC distance we adopt: $\mu_{\mathrm{LMC}}=18.48 \pm 0.03$ mag.

To test the robustness of this technique the analysis was repeated with either one or two data points removed from the input, or with different values for the individual distance

\footnotetext{
8 Use of the Indebetouw et al. (2005) law was justified by Monson et al. (2012).
}

moduli. For example, the data was re-fit with either $K_{s}$, [4.5] or both bands removed. None of these solutions was found to affect the resulting reddening correction or distance modulus at a significant level. We also tested the fit by using the original values from Table 4 of Udalski et al. (2001; with their reddening correction removed). Despite the fact that the change in zeropoint is significant-over $0.2 \mathrm{mag}$ - the resulting fit was barely affected. This shows that the nine-band fit is an excellent way to measure the distance modulus to a population, even if the data is somewhat heterogeneous.

Note that we have made no metallicity correction for the $V$ and $I$ data in this fit. As we will discuss in Section 4, there is some evidence that the zero-points of the optical Leavitt laws are affected by metallicity and there have been many calculations of $\gamma_{W}$, which measures the change in the optical Wesenheit Leavitt law zero-point with changing metallicity. However, $\gamma$ is much more difficult to estimate for the individual optical bands as metallicity effects are degenerate with reddening.

We do not believe that the metallicity effect has a significant effect on the value of $E(B-V)$ derived in this section. The majority of the power in the fit is in the long wavelength range where we have more data, which is where the metallicity effect is believed to be vastly reduced. The small changes induced in $V$ and $I$ due to metallicity effects are much less significant than the corrections we have made to put all the data on the same zero-point.

\section{INDEPENDENT DISTANCE COMPARISONS}

The nine-band fit presented in Figure 6 produces a reddeningcorrected Cepheid distance modulus of $24.29 \pm 0.03_{\text {stat }} \pm$ $0.03_{\text {sys }}$ mag. In this section we compare our result to other recent measurements also using Cepheids, and then compared to other independent distance indicators.

\subsection{Cepheid Comparison}

The Auracaria project is using near-infrared observations of Cepheid populations to determine distances to nearby galaxies. We compare our results with their study of IC 1613 (Pietrzyński et al. 2006), in which the authors use the template fitting method of Soszyński et al. (2005) to obtain mean-light magnitudes of Cepheids in $J$ and $K$ from single-epoch observations. They observed 39 Cepheids in the galaxy, the majority of which are also observed in our study. Adopting the PL slopes from Persson et al. (2004) and adopting an LMC distance modulus of $18.50 \pm 0.10 \mathrm{mag}$ they find $\mu_{J}=24.385 \pm 0.040 \mathrm{mag}$ and $\mu_{K}=24.306 \pm 0.045 \mathrm{mag}$. Both of these values agree with our FourStar results within the $1 \sigma$ uncertainties.

Pietrzyński et al. (2006) combine their $J$ and $K$ distance moduli with the $V$ and $I$ values derived in OGLE II by Udalski et al. (2001) to derive a multi-wavelength fit for $E(B-V)$ and the extinction-corrected distance modulus. Using their fourband fit they measure $E(B-V)=0.090 \pm 0.019 \mathrm{mag}$ and derive $\mu_{0}=24.291 \pm 0.035 \mathrm{mag}$. Their derived reddening is slightly higher than our value of $0.05 \pm 0.01 \mathrm{mag}$, but is barely outside the respective $1 \sigma$ error bars. Their de-reddened distance, however, is in complete agreement with our value of $24.29 \pm 0.03 \mathrm{mag}$. This goes to show the power of moving to the infrared; the effect of reddening is significantly reduced here such that the choice of reddening law and the value of $E(B-V)$ have little effect at these wavelengths.

More recently, Bernard et al. (2010, B10) observed IC 1613 at optical wavelengths using the Advanced Camera for Surveys on 


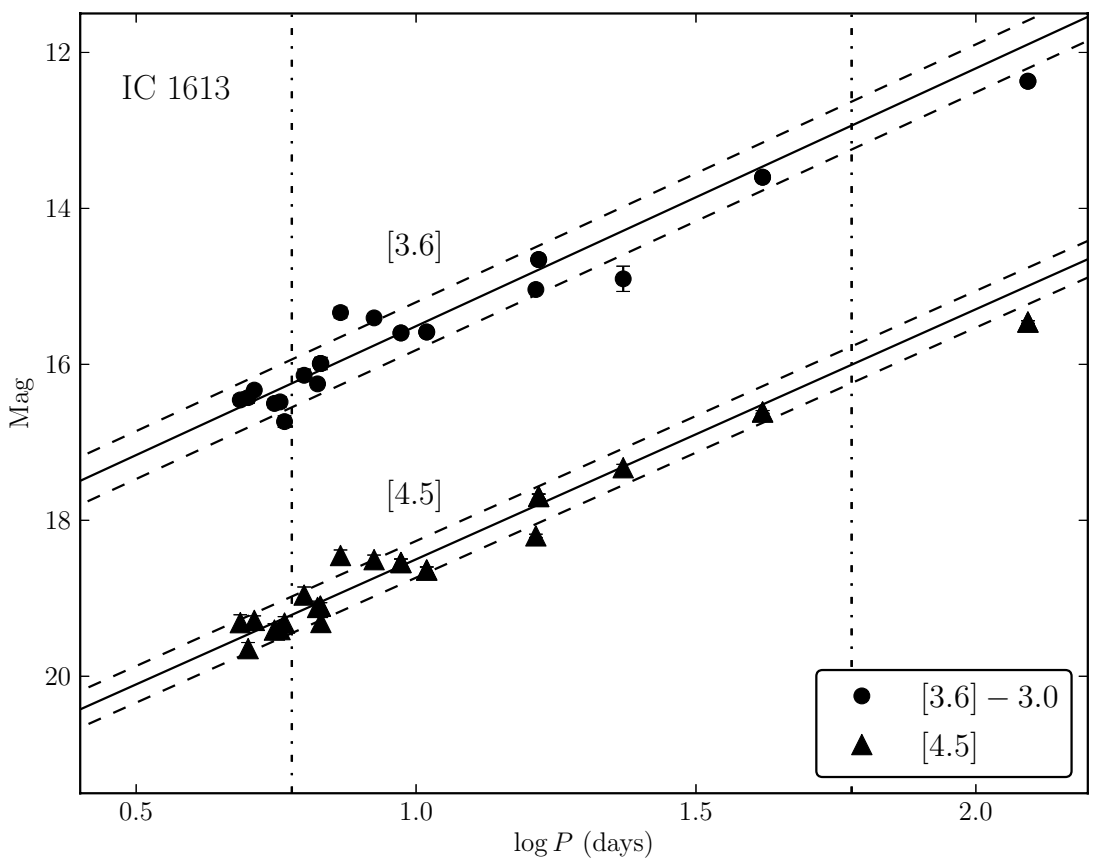

Figure 5. Period-luminosity relations in the 3.6 and $4.5 \mu \mathrm{m}$ bands. The solid lines represent the fitted PL relations; the dashed lines show the $\pm 2 \sigma$ changes in zero-point. The vertical dot-dash lines show the period range (6-60 days) used to fit the PL relations.

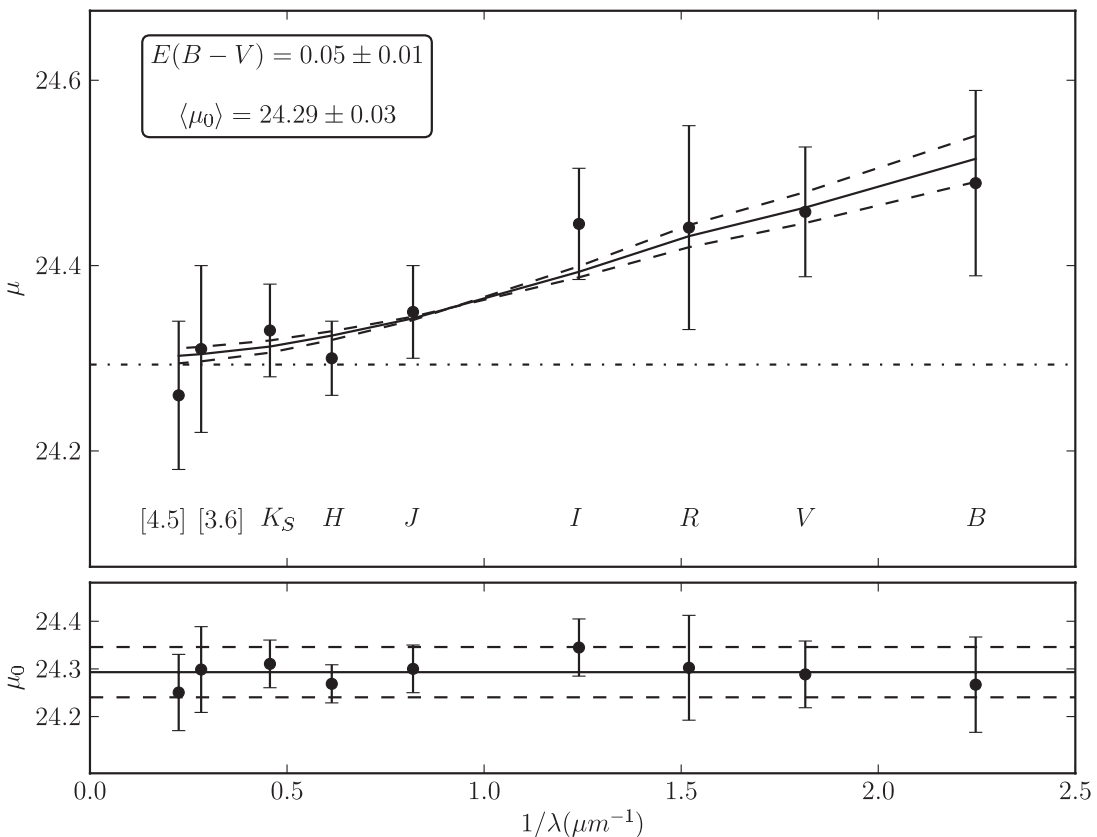

Figure 6. Fitting the IC 1613 distance moduli to the reddening laws of Cardelli et al. (1989; $B$ to $K_{s}$ ) and Indebetouw et al. (2005; $K_{s}$ to [4.5]). Points at $B$ and $R$ are taken from Freedman (1988), $V$ and $I$ are from Udalski et al. (2001), $J, H$ and $K_{s}$ are FourStar data, and [3.6] and [4.5] are IRAC data. The distance moduli from $B$ to $R$ were refit using the LMC PL relations from Fouqué et al. (2007) and adopting $\mu_{\mathrm{LMC}}=18.48$. The solid line is the best-fit reddening law, the dashed lines are $\pm 1 \sigma$ around the law, and the dot-dashed line is the resulting reddening-corrected distance modulus. The bottom panel shows the residuals of the extinction-corrected distance moduli around the mean value.

the Hubble Space Telescope. Their study looked at the fainter variable stars in the galaxy and found 44 Cepheids pulsating in either the fundamental or overtone modes. These Cepheids have short periods (the majority have $\log P<0.5$ ) and were not detected in the CHP observations.

B10 derive the Cepheid distance using the Wesenheit index $W_{V I}$ (Madore 1976). The $W_{V I}$ index is reddening-free by design, hence is only affected by the choice of reddening law, and not at all by the total amount of reddening. Adopting an LMC distance modulus of $18.515 \pm 0.085$ (from Clementini et al. 2003), they find $\mu_{0, W}=24.50 \pm 0.11$ using only the fundamental mode Cepheids. This value is significantly higher than the distance we find in Section 3.3. However, it is possible that this is due to the lack of a metallicity correction on $\mu_{0, W}$.

B10 make no correction for metallicity in their Cepheid analysis, and note that they do not believe a correction is necessary at the low metallicity of IC 1613. It may be the case that the metallicity correction required on $\mu_{0, W}$ decreases as we move to low $[\mathrm{Fe} / \mathrm{H}]$ populations, but this has not been proven conclusively. To this end we take the correction on $\mu_{0, W_{V I}}$ from 


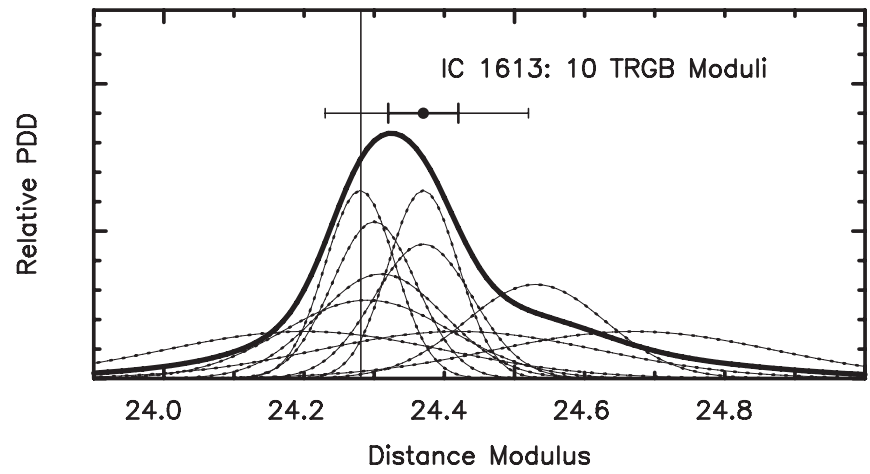

(a)

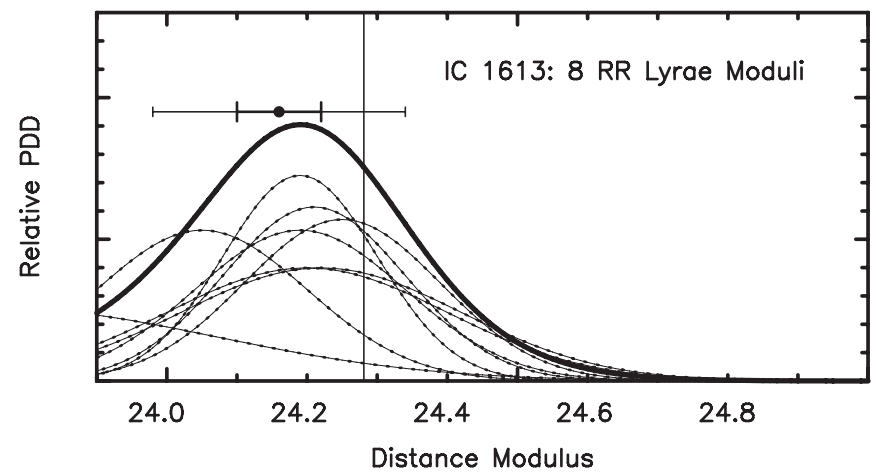

(c)

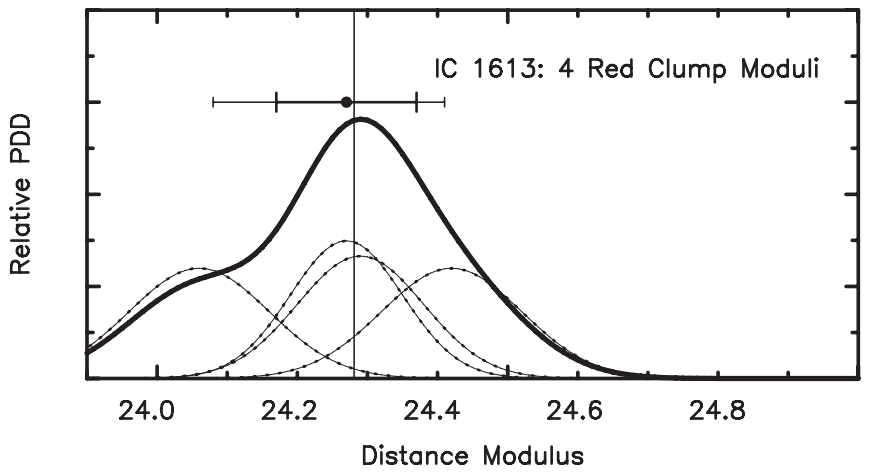

(b)

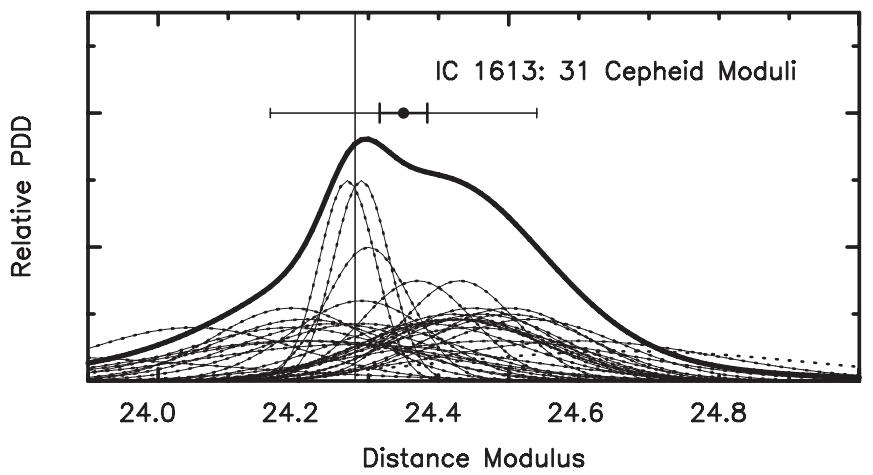

(d)

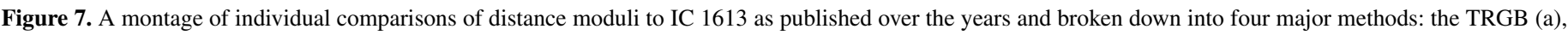

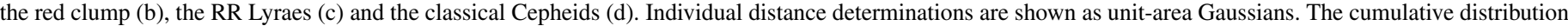

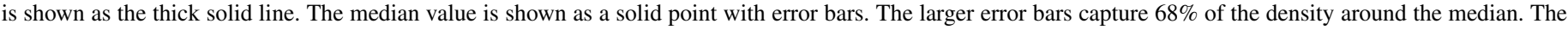
smaller error bar is the error on the mean. For consistent comparison, the vertical solid line marks the Cepheid distance determined in this paper.

Scowcroft et al. (2009) of $\gamma_{W_{V I}}=-0.29 \pm 0.11 \mathrm{mag} \mathrm{dex}^{-1}$ and apply it to the distance derived by B10. Assuming $12+\log (O / H)$ of 8.34 (Sakai et al. 2004) and 7.90 (Bresolin et al. 2007) for the LMC and IC 1613 respectively, and now adopting the CHP LMC distance modulus of $18.48 \mathrm{mag}$, we find a metallicity corrected distance modulus of $\mu_{0, W, Z}=24.33 \pm 0.14$ mag. This is now consistent with our value derived in Section 3.3, but its error bar is much larger than the original B10 result.

We consider the same approach for the optical data from Udalski et al. (2001). Adopting the CHP LMC distance modulus we recalculate their reddening-free distance modulus to be $\mu_{0, W}=24.41 \pm 0.07 \mathrm{mag}$; again, higher than the reddening-corrected distance derived in this paper. Adopting the same metallicity parameters as the previous paragraph we find a reddening-free, metallicity-corrected $\mu_{0, W, Z}=24.24 \pm$ $0.11 \mathrm{mag}$. Like the B10 result, this is consistent with our result, but the metallicity correction has driven up the uncertainty on the value.

It is possible that the large difference between the our distance modulus and that from B10 is due to the very different period distributions of the two samples. B10 focuses on short period $(\log P<0.5)$ Cepheids, while this work studies a longer period sample $(0.77<\log P<1.77)$. The linearity of the Leavitt law in the Wesenheit bands was recently studied by García-Varela et al. (2013). They found that the Wesenheit law may have a break around 10 days, and that metallicity may play a part. It is clear that the metallicity effect on the Leavitt law zero-point in the optical bands requires more study, and that its effect at the lowest metallicities is not yet conclusively ruled out.

\subsection{RR Lyrae Comparison}

The Bernard et al. (2010) study not only found Cepheids in IC 1613 but also RR Lyrae stars. RR Lyrae stars obey a luminosity-metallicity relation at optical wavelengths. Adopting a mean metallicity of $[\mathrm{Fe} / \mathrm{H}]=-1.6 \pm 0.2$ and the luminosity-metallicity relation from Bernard et al. (2009), they calculate the absolute magnitude of the horizontal branch in IC 1613 to be $M_{V}=+0.52 \pm 0.12 \mathrm{mag}$, and derive a reddening corrected RR Lyrae distance modulus of $24.39 \pm 0.12$ mag; but see Section 4.4 and the RR Lyrae comparison panel in Figure 7. This is larger than, but still within $1 \sigma$ of our multi-band Cepheid fit presented above.

\subsection{Tip of the Red Giant Branch Comparison}

An independent measure of the distance to IC 1613 can be obtained using the TRGB. The absolute magnitude of the TRGB is a physical property of the stellar population and does not depend on any measurements further down the distance ladder.

An excellent review of the use of color-magnitude diagram based distance indicators, including the TRGB, is Salaris (2012). Briefly summarized, the $I$ band TRGB is considered a robust distance indicator because the bolometric correction to the $I$ band magnitude as a function of $[\mathrm{Fe} / \mathrm{H}]$ and effective temperature is complementary to the changes in bolometric luminosity due to differences in metallicity. These two effects cancel each other in the $I$ band, meaning that the absolute $I$ band magnitude of the TRGB is essentially constant with both age and metallicity; although see Madore et al. (2009) for their $T$-magnitude technique and calibration of even this small 
residual metallicity effect. This makes the TRGB a robust measure of distance for old resolved stellar populations, and an independent check of the Cepheid distance moduli we have presented in the previous section.

We compare our result to the work of Dolphin et al. (2001, hereafter D01), who derived the TRGB distance to IC 1613 using $V$ and $I$ band photometry from WFPC 2 on Hubble. They provide two estimates of the TRBG apparent magnitude-20.40 \pm 0.09 from their own data and $20.35 \pm 0.07$ from a re-reduction of the data from Cole et al. (1999). The second value is more robust as it is measured from a region with higher stellar density, hence more stars on the red giant branch. They assume the absolute magnitude of the TRGB is $M_{I}=-4.02 \pm 0.05$, and a foreground extinction of $A_{I}=0.05 \pm 0.02$, resulting in an extinction-corrected distance modulus of $\mu_{0}=24.32 \pm$ 0.09 mag.

The true distance modulus derived from our multi-band fit is consistent with the TRGB distance from D01. This suggests that metallicity effects are not significantly affecting the Cepheid distance modulus derived here; our PL relations were all calibrated to the MW and LMC which have much higher average metallicities than IC 1613. Note, however, that this result applies to the Cepheid distance modulus derived from a multi-band fit. It does not necessarily tell us anything about the effect of metallicity on an individual PL relation if reddening and metallicity are covariant/degenerate in selected bandpasses.

\subsection{Dispersion of Independent Measurements}

In Figure 7 we make a graphical comparison of our newly determined Cepheid distance to IC 1613 with the published record of prior distance determinations as found in the 2012 December release of the compilation of redshift-independent distances in NED-D. No attempt has been made to put any of these distances onto a common zero point; the data therefore reflect a variety of adopted reddenings, zero points and wavelengths. We have however, subdivided the data down to a comparison of three major methods: the TRGB method, the RR Lyraes, red clump stars and previous determinations also using Cepheids. The distance modulus determined above is shown as a solid vertical line in each of the plots. Individual determinations are shown as unit-area Gaussians whose width is the published error on the mean. The solid line is the renormalized (Frequentist) sum of those individual probability density distributions (PDDs); its peak is the mode of the published distribution. The filled circle (with horizontal error bars) is the median value of the PDD. The larger error bars capture $68 \%$ of the density around the median. The smaller error bar is the error on the mean.

On average, the (Population II) RR Lyrae variables are seen to give a slightly lower distance moduli than our (Population I) Cepheids. On the other hand, the (Population II) TRGB method appears to give, on average, slightly larger moduli than our Cepheid modulus, although specific studies can be selected that agree exactly. The red clump moduli are few in number and widely scattered, although they do broadly agree with our Cepheid distance. The largest number of distance determinations come from previously published studies of the Cepheids themselves. Here we compare 31 previous determinations and remark that the mode of this distribution is in good agreement with the latest value, although the range of values accumulated over the years is considerable.

In their Figure 16, B10 plot the distance moduli in the literature when corrected to a common LMC distance and common $E(B-V)$. The dispersion is somewhat reduced, but we note that their adopted $\mu_{\mathrm{LMC}}$ is $18.515 \mathrm{mag}$ (compare to the $\mathrm{CHP}$ value of $18.48 \mathrm{mag})$, and their adopted $E(B-V)=0.025 \mathrm{mag}$ is lower than the $E(B-V)=0.05$ mag we derive from our multi-band fit. It is clear from this, and Figure 7 that adopted reddening and the LMC distance are the dominant systematics in the determination of the distance to IC 1613.

Fortunately, reducing these two systematics is entirely the domain of the CHP. Our distance ladder is tied to parallax measurements of MW Cepheids, and we virtually eliminate reddening in the mid-infrared. Our result, $\mu_{0}=24.29 \pm$ $0.03_{\text {stat }} \pm 0.03_{\text {sys }}$ and its quoted errors reflect the reduced systematic uncertainty and increased precision in these values, showing the power of moving to the mid-infrared for Cepheid distance studies.

\subsection{Metallicity Effects in the Mid-infrared}

IC 1613 has a metallicity of $[\mathrm{Fe} / \mathrm{H}] \approx-1$ (D01), significantly lower than the MW and LMC which were the two calibration galaxies for the CHP PL relations. This makes it an ideal testbed for searching for metallicity effects in the Cepheid PL. Initial tests for metallicity sensitivity in the mid-infrared were presented in Freedman et al. (2012), where we plotted the residual from the PL relation against spectroscopic metallicity for individual Cepheids. We found no significant effect at $-0.6 \leqslant[\mathrm{Fe} / \mathrm{H}] \leqslant 0.2$. Including IC 1613 in our studies increases the metallicity range of CHP Cepheids by a factor of two, so if a significant metallicity effect were present we should be able to detect it somewhere in this range.

To test for a metallicity effect in IC 1613 we must use a different tactic from the one we applied in the MW and LMC. As we do not have metallicity measurements of the individual stars we must treat them as an ensemble. We assume that there is no effect on the PL slope and that any difference would manifest itself in the zero-point. Therefore, if composition does have an effect, we should find a different distance modulus than with an independent measure such as the TRGB or red clump.

Correcting our [3.6] and [4.5] distances for extinction using $E(B-V)=0.05 \pm 0.01 \mathrm{mag}$ we derive $\mu_{0,[3.6]}=24.30 \pm$ 0.09 mag and $\mu_{0,[4.5]}=24.25 \pm 0.08$ mag, respectively. Both of these values are in excellent agreement with the TRGB distance from D01. The $4.5 \mu \mathrm{m}$ distance modulus is slightly (0.05 mag) brighter than $\mu_{0,[3.6]}$ but still agrees to within $1 \sigma$. As has been discussed previously (Freedman et al. 2011; Scowcroft et al. 2011; Monson et al. 2012), we believe that the [4.5] band is unsuitable for distance measurements as it is contaminated by the temperature and metallicity sensitive $\mathrm{CO}$ band-head at $4.6 \mu \mathrm{m}$. Henceforth, all references to our mid-infrared Cepheid distance pertain solely to the $3.6 \mu \mathrm{m}$ measurement.

The consistency of our [3.6] distance modulus with the TRGB, red clump and multi-band distances shows again that metallicity is not significantly impacting the distance measurements over the range $-1.0 \leqslant[\mathrm{Fe} / \mathrm{H}] \leqslant 0.2$. Therefore we conclude that there is no effect of metallicity on the $3.6 \mu \mathrm{m}$ Cepheid PL relation zero-point at the level of \pm 0.09 mag. The mid-infrared measurement of the Hubble constant needs no adjustment for metallicity effects.

\section{SUMMARY AND CONCLUSIONS}

We performed a multi-epoch survey of IC 1613 using Spitzer in the mid-infrared and the new FourStar camera on Magellan in the near-infrared. The photometric catalogs were matched to the OGLE Cepheid catalog to locate the Cepheids. 
Mean-light magnitudes were obtained for each star and PL relations were constructed in the $J, H, K_{s}$, [3.6] and [4.5] bands, from which distance moduli were derived. Using the $3.6 \mu \mathrm{m}$ PL relation, where the effects of reddening are minimized, we measure the true distance modulus of IC 1613 as $\mu_{0,3.6}=24.30 \pm 0.09_{\text {stat }} \pm 0.03_{\text {sys }}$ mag. This is entirely consistent with the independent TRGB and red clump distance moduli derived in Dolphin et al. (2001).

In addition to the single-band mid-infrared distance we have used near-infrared data from FourStar and archival optical data (corrected to an LMC distance of $\mu_{\mathrm{LMC}}=18.48 \pm 0.03 \mathrm{mag}$ ) to derive a nine-band fit to measure the reddening and distance modulus of IC 1613. We find $E(B-V)=0.05 \pm 0.01$ and $\mu_{0}=24.29 \pm 0.03_{\text {stat }} \pm 0.03_{\text {sys }}$ mag.

Finally, we have shown that as the mid-infrared Cepheid distance agrees with the TRGB distance, there must be no significant metallicity effect on the PL relation in the range $-1.0 \leqslant[\mathrm{Fe} / \mathrm{H}] \leqslant 0.2$. This removes any uncertainty in the CHP distance scale due to metallicity effects in the Cepheid calibration, significantly reducing the uncertainty in the CHP mid-infrared determination of the Hubble constant.

We thank the referee for their thoughtful response, which improved the content and style of the paper.

This research made use of the NASA/IPAC Extragalactic database (NED) which is operated by the Jet Propulsion Laboratory, California Institute of Technology, under contract with the National Aeronautics and Space Administration.

This research made use of APLpy, an open-source plotting package for Python hosted at http://aplpy.github.com

Facilities: Spitzer, Magellan:Baade

\section{APPENDIX}

\section{FITTING TEMPLATE LIGHT CURVES}

The amplitudes of Cepheid light curves in the near-infrared are much smaller than at optical wavelengths (approximately $1 / 3$ to $2 / 3$ of the $V$ or $I$ band amplitudes-see Table 2 of Soszyński et al. 2005). However, they still reach levels around 0.5 mag which, combined with non-uniform sampling, can significantly affect the mean-light magnitude derived from a straight average. The effect of small numbers of non-uniform observations can be negated using template fitting, as was first shown by Freedman (1988) and later elaborated upon by Soszyński et al. (2005). In that paper they made template light curves in the $J, H$, and $K_{s}$ bands that could be scaled and phased using a complete $V$ or $I$ band light curve and a single nearinfrared observation.

The technique was used by the Araucaria project to derive higher accuracy mean-light magnitudes from single observations in the $J$ and $K$ bands. They successfully applied the method to Cepheids in IC 1613 (Pietrzyński et al. 2006), obtaining PL relations in each band from either one or two observations per star. Here we test this method to derive mean-light magnitudes for our near-infrared Cepheids.

The most fundamental piece of information in the template fitting technique is the period of the Cepheid. From this and the $V$ or $I$ light curve the time of maximum light in the reference (optical) band is predicted. The phase-lag between the reference band and the near-infrared band is known and can be used to predict the time of maximum light in each of the near-infrared bands. The amplitude of the light curve is scaled to the amplitude of the reference light curve. The template is then fit to each near-
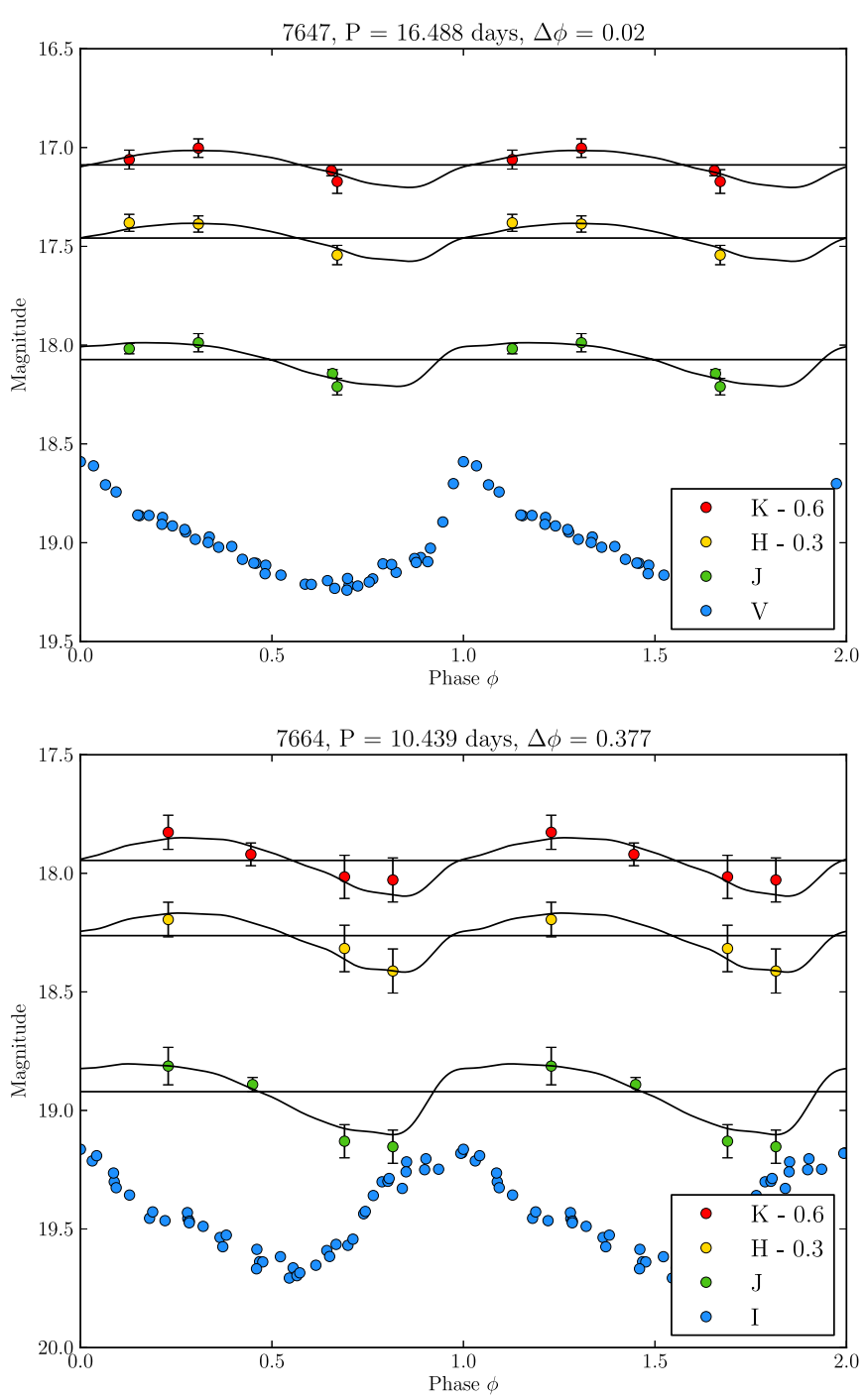

Figure 8. Light curves fit using the template method from Soszyński et al. (2005). A phase shift, $\Delta \phi$ was incorporated into the algorithm to account for possible period changes, and hence deviations from the predicted time of maximum light in the near-infrared bands. The $J$ and $K_{s}$ light curves also contain points from Pietrzyński et al. (2006) where available. The Cepheid IDs use the OGLE numbering scheme.

(A color version of this figure is available in the online journal.)

infrared observation individually and the mean-light magnitude is calculated; a weighted mean of these values gives the best estimate of the mean-light magnitude of the Cepheid.

It is imperative to have highly precise periods for the Cepheids; if the time of maximum light is computed incorrectly then the relative phases of each data point will be erroneous and the mean-light magnitude will be incorrect. As the observations in Pietrzyński et al. (2006) were taken several years after the data for the reference light curves they took three more $V$ band observations contemporaneously with the near-infrared data. This allowed them to refine the periods and define the time of maximum light $(\phi=0)$ more accurately. The periods typically changed by $0.1 \%-0.5 \%$, but over $10 \mathrm{yr}$ this $\Delta P$ is sufficient to shift the time of maximum light by as much as $\phi= \pm 0.5$ compared to the original estimate.

We do not have contemporaneous optical data to further refine the periods. We adopted the periods from Pietrzyński et al. (2006) where they were available and Udalski et al. (2001) in all other cases. To account for the less accurate periods the fitting 
algorithm was altered to allow for a phase shift. The best-fit phase shift was calculated by stepping through the possible shifts with a step size of $\delta \phi=0.001$ and minimizing the residuals of the points around the template light curve. Example light curves for two Cepheids are shown in Figure 8. The phase shifts were found to be anywhere in the range $-0.4 \leqslant \Delta \phi \leqslant 0.4$. To reiterate, shifts of this size could be induced by a period change of less than $1 \%$ over $10 \mathrm{yr}$. At this point it is clear that we do not know the periods of the Cepheids well enough to predict the time of maximum light to the required degree of accuracy, and so cannot determine the phase of any of our data points with a high degree of confidence.

To confirm these thoughts the PL relations were plotted using the template mean-light magnitudes. The resulting apparent moduli showed marginal changes - at the level of $1 \sigma$ - but no significant differences.

We conclude that although our knowledge of the periods of the Cepheids is good enough to derive a PL relation, it is not sufficient for determining the time of maximum light to the accuracy required for the template fitting technique. For the rest of this work we adopt the regular mean values for $J, H$, and $K_{s}$ as listed in Table 3.

\section{REFERENCES}

Baade, W. 1963, in Evolution of Stars and Galaxies, ed. C. H. Payne-Gaposhkin (Cambridge: Harvard Univ. Press), 218

Bernard, E. J., Monelli, M., Gallart, C., et al. 2009, ApJ, 699, 1742

Bernard, E. J., Monelli, M., Gallart, C., et al. 2010, ApJ, 712, 1259 (B10)

Bresolin, F., Urbaneja, M. A., Gieren, W., Pietrzyński, G., \& Kudritzki, R.-P. 2007, ApJ, 671, 2028

Cardelli, J. A., Clayton, G. C., \& Mathis, J. S. 1989, ApJ, 345, 245

Carpenter, J. M. 2001, AJ, 121, 2851

Clementini, G., Gratton, R., Bragaglia, A., et al. 2003, AJ, 125, 1309

Cole, A. A., Tolstoy, E., Gallagher, J. S., III, et al. 1999, AJ, 118, 1657

de Vaucouleurs, G., de Vaucouleurs, A., Corwin, H. G., Jr., et al. 1991, in Third Reference Catalogue of Bright Galaxies, ed. N. G. Roman, G. de Vaucouleurs, A. de Vaucouleurs, H. G. Corwin, Jr., R. J. Buta, G. Paturel, \& P. Fouqué (New York: Springer), vol. 11, 77
Dolphin, A. E., Saha, A., Skillman, E. D., et al. 2001, ApJ, 550, 554

Fouqué, P., Arriagada, P., Storm, J., et al. 2007, A\&A, 476, 73

Freedman, W. L. 1988, ApJ, 326, 691

Freedman, W. L., \& Madore, B. F. 1990, ApJ, 365, 186

Freedman, W. L., Madore, B. F., Scowcroft, V., et al. 2011, AJ, 142, 192

Freedman, W. L., Madore, B. F., Scowcroft, V., et al. 2012, ApJ, 758, 24

Freedman, W. L., Rigby, J., Madore, B. F., et al. 2009, ApJ, 695, 996

García-Varela, A., Sabogal, B. E., \& Ramírez-Tannus, M. C. 2013, MNRAS, 431, 2278

Indebetouw, R., Mathis, J. S., Babler, B. L., et al. 2005, ApJ, 619, 931

Kennicutt, R. C., Jr., Stetson, P. B., Saha, A., et al. 1998, ApJ, 498, 181

Lee, M. G., Freedman, W. L., \& Madore, B. F. 1993, ApJ, 417, 553

Madore, B. F. 1976, in Royal Greenwich Observatory Bulletins, Vol. 182, The Galaxy and the Local Group, ed. R. J. Dickens, J. E. Perry, F. G. Smith, \& I. R. King (Herstmonceaux: Royal Greenwich Observatory), 153

Madore, B. F., \& Freedman, W. L. 2012, ApJ, 744, 132

Madore, B. F., Mager, V., \& Freedman, W. L. 2009, ApJ, 690, 389

Makovoz, D., \& Khan, I. 2005, in ASP Conf. Ser. 347, Astronomical Data Analysis Software and Systems XIV, ed. P. Shopbell, M. Britton, \& R. Ebert (San Francisco, CA: ASP), 81

McGonegal, R., McAlary, C. W., Madore, B. F., \& McLaren, R. A. 1982, ApJL, 257, L33

Monson, A. J., Freedman, W. L., Madore, B. F., et al. 2012, ApJ, 759,146

Persson, S. E., Madore, B. F., Krzemiński, W., et al. 2004, AJ, 128, 2239

Persson, S. E., Murphy, D. C., Smee, S., et al. 2013, PASP, 125, 654

Pietrzyński, G., Gieren, W., Soszyński, I., et al. 2006, ApJ, 642, 216

Reach, W. T., Megeath, S. T., Cohen, M., et al. 2005, PASP, 117, 978

Romaniello, M., Primas, F., Mottini, M., et al. 2008, A\&A, 488, 731

Sakai, S., Ferrarese, L., Kennicutt, R. C., Jr., \& Saha, A. 2004, ApJ, 608,42

Salaris, M. 2012, Ap\&SS, 341, 65

Sandage, A. 1971, ApJ, 166, 13

Scowcroft, V., Bersier, D., Mould, J. R., \& Wood, P. R. 2009, MNRAS, 396, 1287

Scowcroft, V., Freedman, W. L., Madore, B. F., et al. 2011, ApJ, 743, 76

Skrutskie, M. F., Cutri, R. M., Stiening, R., et al. 2006, AJ, 131, 1163

Soszyński, I., Gieren, W., \& Pietrzyński, G. 2005, PASP, 117, 823

Stetson, P. B. 1987, PASP, 99, 191

Stetson, P. B. 1994, PASP, 106, 250

Stetson, P. B., \& Harris, W. E. 1988, AJ, 96, 909

Storm, J., Gieren, W., Fouqué, P., et al. 2011, A\&A, 534, A95

Udalski, A., Wyrzykowski, L., Pietrzynski, G., et al. 2001, AcA, 51, 221

Wolf, M. 1906, MNRAS, 67, 91 